\title{
Shear-induced migration in flowing polymer solutions: Simulation of long-chain DNA in microchannels
}

\author{
Richard M. Jendrejack ${ }^{\mathrm{a})}$ \\ Department of Chemical and Biological Engineering, University of Wisconsin-Madison, \\ Madison, Wisconsin 53706 \\ David C. Schwartz \\ Department of Chemistry and Laboratory of Genetics, Laboratory for Molecular and Computational \\ Genomics, University of Wisconsin-Madison, Madison, Wisconsin 53706 \\ Juan J. de Pablob) and Michael D. Graham ${ }^{\text {c) }}$ \\ Department of Chemical and Biological Engineering, University of Wisconsin-Madison, \\ Madison, Wisconsin 53706
}

(Received 30 April 2003; accepted 5 November 2003; publisher error corrected 24 February 2004)

\begin{abstract}
We simulate dilute solution dynamics of long flexible polymer molecules in pressure driven flow in channels with widths of roughly $0.1-10$ times the polymer bulk radius of gyration. This is done using a self-consistent coarse-grained Langevin description of the polymer dynamics and a numerical simulation of the flow in the confined geometry that is generated by the motions of polymer segments. Results are presented for a model of DNA molecules of $\sim 10-100 \mu \mathrm{m}$ contour length in micron-scale channels. During flow, the chains migrate toward the channel centerline, in agreement with well-known experimental observations. The thickness of the resulting hydrodynamic depletion layer increases with molecular weight at constant flow strength; higher molecular weight chains therefore move with a higher average axial velocity than lower molecular weight chains. In contrast, if the hydrodynamic effects of the confining geometry are neglected, depletion of concentration is observed in the center of the channel rather than at the walls, contradicting experimental observations. The mechanisms for migration are illustrated using a simple kinetic theory dumbbell model of a confined flexible polymer. The simple theory correctly predicts the trends observed in the detailed simulations. We also examine the steady-state stretch of DNA chains as a function of channel width and flow strength. The flow strength needed to stretch a highly confined chain away from its equilibrium length is shown to increase with decreasing channel width, independent of molecular weight; this is fairly well explained using a simple blob picture. (C) 2004 American Institute of Physics. [DOI: 10.1063/1.1637331]
\end{abstract}

\section{INTRODUCTION}

New and developing technologies for single molecule manipulation and analysis of DNA in micron and nanometer scale devices ${ }^{1-6}$ have fueled considerable interest in the structure and dynamics of solutions of DNA in confined geometries. Predictive methods capable of describing the conformation and motion of polymer chains in micro- and nanofluidic geometries would be of considerable significance for the conception and design of such devices. In this work, we investigate the dynamics of DNA molecules of contour length $10.5-126 \mu \mathrm{m}$; the free-solution radii and relaxation times (in a $1 \mathrm{cP}$ solvent) of these molecules are $\approx 0.5-2 \mu \mathrm{m}$ and $\approx 0.03-2.4 \mathrm{~s}$, respectively. The long-time dynamics of these DNA molecules are out of reach of atomistic simulations. However, they are accessible to coarse-grained, Langevin models. ${ }^{7,8}$ The central challenge is to consider polymer and solvent motion simultaneously and self-consistently,

\footnotetext{
a)Permanent address: 3M Corporation, St. Paul, MN 55144-1000.

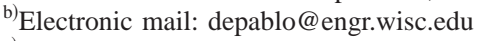

c) Author to whom correspondence should be addressed. Electronic mail: graham@engr.wisc.edu
}

with the solvent motion satisfying the no-slip boundary condition on the surfaces of the confining geometry. We have previously developed a computationally tractable formalism to address this challenge, and used it to investigate relaxation and diffusion of DNA in micron-scale channels. ${ }^{9}$ Here, we apply the method to generate the first predictions of the pressure-driven flow behavior of long DNA molecules in channels of micrometer dimensions. Hydrodynamic interactions in confined, flowing systems are shown to give rise to a pronounced, molecular-weight-dependent depletion layer at the walls, thereby providing a basis for separation processes for DNA. As we will show, the depletion layer arises from polymer-wall hydrodynamic interaction.

Molecular migration in flowing dilute polymeric solutions is a well-known phenomenon that has received a fair amount of experimental and theoretical investigation. Much of this was recently reviewed by Agarwal et al. ${ }^{10}$ who examined various experimental and theoretical studies and concluded:

We find a disagreement between different theories, even with regard to their prediction of the direction of polymer migration during simple flows. While we have seen that 
these mechanisms can explain many indirect implications of the flow-induced polymer migration phenomena, they are able to explain only some and not all of the direct experimental results, even qualitatively.

Spatial variations in polymer concentration arise from symmetry-breaking contributions to the steady-state mass flux of polymer. In inhomogeneous rectilinear channel flow of dilute linear polymers, general considerations from polymer kinetic theory point to two sources of migration:

(a) coupling of hydrodynamics with spatial variations in the polymer conformational distribution function lead to Brownian migration, ${ }^{10-13}$ and

(b) in the presence of a wall, symmetry of the hydrodynamic interaction is broken, leading to deterministic migration. ${ }^{14}$

The deterministic contribution to migration is present even in homogeneous flows such as simple shear near a wall. In inhomogeneous flows, the wall hydrodynamic interaction also contributes additional terms to the Brownian migration. Note that if one ignores wall contributions, the point-force (Oseen-Burgers) hydrodynamic interaction is anisotropic but symmetric, and deterministic migration is not predicted. The above discussion assumes that the hydrodynamics of the polymer molecule are adequately represented by a chain of point forces. Past studies ${ }^{10-13}$ have almost universally neglected wall hydrodynamic interaction. The kinetic theory predictions for migration in the absence of wall hydrodynamic interaction have been mixed. For example, the use of preaveraged bulk hydrodynamic interactions for a polymer in a parabolic velocity profile predicts a weak depletion of polymer at the wall, while using nonpreaveraged bulk hydrodynamic interactions predicts depletion at the center line of the channel. Jhon and Freed ${ }^{14}$ used a kinetic theory dumbbell model of a polymer near an infinite plane wall with approximate wall hydrodynamic interactions and concluded that this contribution to migration was away from the wall. In Sec. IV of the present work, we review and extend these kinetic theory results. Turning now to direct simulations, Fan et al. ${ }^{15}$ used dissipative particle dynamics (DPD) ${ }^{16,17}$ to study the behavior of flexible polymers in rectilinear flow through microchannels, and predicted very weak hydrodynamic depletion at the walls. However, for their choice of DPD parameters, diffusion was artificially fast relative to momentum transport; the Schmidt number for the solvent was $O(1)$. In this situation, a Schmidt number of $O(1)$, hydrodynamic interactions are still developing on the time scale of polymer motion, and the consequences of this for the polymer dynamics is yet unknown. ${ }^{18}$ In any event, the DPD results appear rather different than the results presented in this work, which do not suffer from the Schmidt number problem and indicate a significant depletion of polymer near the walls.

Migration is also observed for solutions of rigid Brownian fibers in inhomogeneous flows. Recent theoretical studies by Nitsche and Hinch $^{19}$ as well as Schiek and Shaqfeh ${ }^{20}$ predict weak migration toward the walls for (rectilinear) parabolic flow between flat plates; wall hydrodynamic interaction and steric effects were not considered. Earlier, de
Pablo and Öttinger ${ }^{21}$ had considered steric effects but not hydrodynamic interactions and found a decrease in the thickness of the static wall depletion layer-again, migration toward the wall.

Finally, deterministic migration of droplets immersed in flowing fluids has also received a fair amount of attention. ${ }^{22}$ Starting with a rigid particle in a Newtonian fluid at zero Reynolds number, Chan and Leal ${ }^{23}$ systematically examined the perturbative effects of inertia, droplet deformability, and non-Newtonian fluid character. For a slightly deformable drop in a Newtonian fluid in zero Reynolds simple shear flow near a wall, wall hydrodynamic interaction is the sole contribution to droplet migration. Chan and Leal, using farfield wall hydrodynamic interaction, found that drift was always away from the wall (in agreement with experiment). For inhomogeneous flows, they found that the first order contribution was interaction with the gradient of the local shear rate, provided the shear rate changed significantly over the length scale of the droplet. They found that the direction of migration depended on the ratio of solvent and droplet viscosities; for the range of viscosity ratios used in experiments, migration was always toward regions of lower local shear rate (toward the "center"). For circular Couette flow, they found that the final position of the droplet was determined from a competition between the streamline curvature effect and wall hydrodynamic interaction. Related to droplet migration in unbounded inhomogeneous flows are the deterministic studies on migration of nearly rigid cyclic bead spring models ${ }^{24}$ and the dumbbell model of Brunn, ${ }^{25}$ which treated the beads as solid finite-sized spheres. Those model particles are predicted to migrate deterministically toward regions of lower local shear rate in unbounded (no wall hydrodynamic interaction) inhomogeneous flows.

In this work, we present Brownian dynamics simulation results for dilute solution behavior of DNA in pressuredriven flow through square micron-scale channels, accounting for hydrodynamic interactions in the confining geometry, steric effects, and excluded volume (good solvent) interactions. Migration of DNA is a central theme of this study, and in Sec. IV we use kinetic theory for a simple dumbbell model to elucidate the various mechanisms contributing to variations in the steady-state concentration of polymer mass.

\section{MODEL AND SIMULATION APPROACH FOR CONFINED POLYMER DYNAMICS}

\section{A. Bulk solution model}

In the present work, we extend to the microchannel situation a coarse-grained molecular model and simulation method that we have previously shown to provide an accurate representation of structural and dynamical experimental data for DNA in bulk solution with high salt concentration. $^{26,27}$ A linear molecule dissolved in a viscous solvent is represented by $N_{b}$ interaction sites (beads) connected through $N_{s}=N_{b}-1$ entropic connectors (springs). A force balance on this chain leads to the stochastic differential equation $^{7,26-28}$ 


$$
\begin{aligned}
& d \mathbf{r}=\left[\mathbf{u}+\frac{1}{k_{B} T} \mathbf{D} \cdot \mathbf{f}+\frac{\partial}{\partial \mathbf{r}} \cdot \mathbf{D}\right] d t+\sqrt{2} \mathbf{B} \cdot d \mathbf{w}, \\
& \mathbf{D}=\mathbf{B} \cdot \mathbf{B}^{T}
\end{aligned}
$$

Here $k_{B}$ is Boltzmann's constant and $T$ is the absolute temperature. The vector $\mathbf{r}$ contains the $3 N_{b}$ spatial coordinates of the beads that constitute the polymer chain, with $\mathbf{r}_{i}$ denoting the three Cartesian coordinates of bead $i$. The vector $\mathbf{f}$ has length $3 N_{b}$, with $\mathbf{f}_{i}$ denoting the total non-Brownian, nonhydrodynamic force acting on bead $i$. The vector $\mathbf{u}$ of length $3 N_{b}$ represents the unperturbed velocity field (i.e., the velocity field in the absence of the polymer), with $\mathbf{u}_{i}=\mathbf{v}\left(\mathbf{r}_{i}\right)$ denoting the unperturbed velocity field at the location of bead $i$. The components of $d \mathbf{w}$ are obtained from a real-valued Gaussian distribution with mean zero and variance $d t$.

The motion of a segment of the macromolecule creates a velocity field in the fluid, which in turn affects the motion of the entire macromolecule. The velocity field generated by motion of the macromolecule is taken to be due to a chain of point forces acting on the fluid. These hydrodynamic interactions (HI) enter the chain dynamics through the $3 \times 3$ block components of the $3 N_{b} \times 3 N_{b}$ diffusion tensor, D, which can be expressed as

$$
\mathbf{D}_{i j}=k_{B} T\left(\frac{1}{6 \pi \eta a} \mathbf{I} \delta_{i j}+\boldsymbol{\Omega}_{i j}\right),
$$

where $\eta$ is the solvent viscosity, $a$ is the bead hydrodynamic radius, and $\boldsymbol{\Omega}_{i j}$ is the hydrodynomic interaction tensor, ${ }^{7,29}$ which relates the velocity perturbation at point $\mathbf{r}_{i}$ to a point force at $\mathbf{r}_{j}$. The Brownian forces, ${ }^{30}$ which appear in the last term of Eq. (1), are intimately coupled to these velocity perturbations through the fluctuation-dissipation theorem [Eq. (2)].

In an infinite domain (no walls), the Stokes flow velocity field due to a point force located at $\mathbf{x}_{j}$ is given by $\mathbf{v}^{\prime}(\mathbf{x})$ $=\boldsymbol{\Omega}^{\mathrm{OB}}\left(\mathbf{x}-\mathbf{x}_{j}\right) \cdot \mathbf{f}\left(\mathbf{x}_{j}\right)$, where $\boldsymbol{\Omega}^{\mathrm{OB}}$ is the free-space Green's function, or Oseen-Burgers tensor ${ }^{7,29}$

$$
\mathbf{\Omega}^{\mathrm{OB}}\left(\mathbf{x}-\mathbf{x}_{j}\right)=\frac{1}{8 \pi \eta\left|\mathbf{x}-\mathbf{x}_{j}\right|}\left[\mathbf{I}+\frac{\left(\mathbf{x}-\mathbf{x}_{i}\right)\left(\mathbf{x}-\mathbf{x}_{i}\right)}{\left|\mathbf{x}-\mathbf{x}_{j}\right|^{2}}\right] .
$$

The tensor, $\boldsymbol{\Omega}_{i j}$, appearing in Eq. (3) is then given by

$$
\boldsymbol{\Omega}_{i j}=\left(1-\delta_{i j}\right) \boldsymbol{\Omega}^{\mathrm{OB}}\left(\mathbf{r}_{i}-\mathbf{r}_{j}\right) .
$$

In Brownian dynamics simulations ${ }^{26,27,31}$ of polymers, $\boldsymbol{\Omega}^{\mathrm{OB}}$ is typically replaced with the Rotne-Prager-Yamakawa (RPY) tensor ${ }^{32}$

$$
\begin{aligned}
\mathbf{\Omega}^{\mathrm{RPY}}\left(\mathbf{x}-\mathbf{x}_{j}\right) \\
=\frac{1}{8 \pi \eta\left|\mathbf{x}-\mathbf{x}_{j}\right|}\left[C_{1} \mathbf{I}+C_{2} \frac{\left(\mathbf{x}-\mathbf{x}_{i}\right)\left(\mathbf{x}-\mathbf{x}_{i}\right)}{\left|\mathbf{x}-\mathbf{x}_{j}\right|^{2}}\right] \\
\quad \text { if }\left|\mathbf{x}-\mathbf{x}_{j}\right| \geqslant 2 a, \\
=\frac{1}{6 \pi \eta a}\left[C_{1}^{\prime} \mathbf{I}+C_{2}^{\prime} \frac{\left(\mathbf{x}-\mathbf{x}_{i}\right)\left(\mathbf{x}-\mathbf{x}_{i}\right)}{\left|\mathbf{x}-\mathbf{x}_{j}\right|^{2}}\right] \text { if }\left|\mathbf{x}-\mathbf{x}_{j}\right|<2 a,
\end{aligned}
$$

$$
\begin{aligned}
& C_{1}=1+\frac{2 a^{2}}{3\left|\mathbf{x}-\mathbf{x}_{j}\right|^{2}}, \\
& C_{2}=1-\frac{2 a^{2}}{\left|\mathbf{x}-\mathbf{x}_{j}\right|^{2}}, \\
& C_{1}^{\prime}=1-\frac{9\left|\mathbf{x}-\mathbf{x}_{j}\right|}{32 a}, \\
& C_{2}^{\prime}=\frac{3\left|\mathbf{x}-\mathbf{x}_{j}\right|}{32 a} .
\end{aligned}
$$

A diffusion tensor generated using RPY hydrodynamics is guaranteed to be positive-semidefinite for all chain configurations (it is positive definite unless $\mathbf{x}_{i}=\mathbf{x}_{j}$ for some pair of beads). For Oseen-Burgers and RPY hydrodynamics, $\boldsymbol{\Omega}_{i j}$ $=0$ for $i=j$, and $\partial / \partial \mathbf{r}_{i} \cdot \boldsymbol{\Omega}_{i j}=0$ for all $i, j$. In Sec. II B we describe how the above discussion is modified for bounded domains.

The spring force between adjacent beads is described by means of a worm-like spring model ${ }^{33,34}$ which has been shown to be appropriate for molecules such as DNA having stiff backbones $27,31,34-39$

$$
\mathbf{f}_{i j}^{s}=\frac{k_{B} T}{2 b_{k}}\left[\left(1-\frac{\left|\mathbf{r}_{j}-\mathbf{r}_{i}\right|}{q_{o}}\right)^{-2}-1+\frac{4\left|\mathbf{r}_{j}-\mathbf{r}_{i}\right|}{q_{o}}\right] \frac{\mathbf{r}_{j}-\mathbf{r}_{i}}{\left|\mathbf{r}_{j}-\mathbf{r}_{i}\right|} .
$$

Here $\mathbf{f}_{i j}^{s}$ is the force exerted on bead $i$ due to connectivity with bead $j$, and $b_{k}$ is the Kuhn length of the molecule. Letting $N_{k}$ represent the number of Kuhn segments in the molecule and $N_{k, s}$ the number of Kuhn segments per spring, $q_{o}=N_{k, s} b_{k}$ is the maximum spring length, or the contour length of the portion of the molecule represented by one spring. The contour length of the chain is then given by $L$ $=N_{s} q_{o}$.

Following our previous work, ${ }^{27,31,35}$ for the excluded volume potential between two beads of the chain, we use

$$
U_{i j}^{\mathrm{ev}}=\frac{1}{2} v k_{B} T N_{k, s}^{2}\left(\frac{3}{4 \pi S_{s}^{2}}\right)^{3 / 2} \exp \left[\frac{-3\left|\mathbf{r}_{j}-\mathbf{r}_{i}\right|^{2}}{4 S_{s}^{2}}\right],
$$

where $v$ is the excluded volume parameter, and $S_{s}^{2}$ $=N_{k, s} b_{k}^{2} / 6$ is the radius of gyration of an ideal chain consisting of $N_{k, s}$ Kuhn segments.

Using $N_{s}=10$ for $21 \mu \mathrm{m}$ stained $\lambda$-phage DNA at room temperature in a $43.3 \mathrm{cP}$ solvent, in previous work ${ }^{27,35}$ we determined, by direct comparison to available bulk experimental data, that suitable parameter values are $b_{k}$ $=0.106 \mu \mathrm{m}, \quad a=0.077 \mu \mathrm{m}$, and $v=0.0012 \mu \mathrm{m}^{3}$. With these values, the model outlined above was able to reproduce the experimentally observed bulk relaxation time, diffusivity, and equilibrium stretch (size) of DNA. The model also gives results in quantitative agreement with transient and steadystate behavior of $21 \mu \mathrm{m}$ DNA in both simple shear and planar extension over a wide range of Weissenberg numbers. In contrast to other available treatments for DNA in solution, the model discussed here also produces diffusivity results in quantitative agreement with experimental data for chains ranging from 21 to $126 \mu \mathrm{m}$, underscoring its predictive capability. After appropriate modifications to account for confinement, as detailed below, the model should provide useful predictions of DNA behavior in microfluidic devices. 


\section{B. Confinement}

As mentioned above, simple arguments predict that the dynamics of a dissolved macromolecule confined to a channel comparable to its equilibrium coil size $(\sim 1 \mu \mathrm{m}$ for viral DNA) are different from those in free solution, largely as a consequence of the no-slip boundary condition on the fluid motion. Available analytical work ${ }^{29,40,41}$ has concentrated on resolving particle-wall interactions for a few special geometries (e.g., a spherical particle near an infinite plane, between two infinite planes, or at the center line of a cylindrical channel). The long-range effect of a single wall on the mobility of a particle decays slowly as $1 / h$, where $h$ is the distance from the wall. Hydrodynamic interactions between confined particles decay as $1 / r^{2}$ for particles near an infinite plane and exponentially in a channel. ${ }^{29,41}$ Dufresne et al. ${ }^{42}$ recently considered the hydrodynamic coupling of two Brownian particles near a plane wall. Those authors compared their experimental results to analytical predictions for point particles near an infinite plane-i.e., keeping only the leading order far field effects-and found good agreement with experimental observation provided $(h / a)>2$, where $a$ is the radius of the particle. That study represents the first direct experimental validation of the use of point particle hydrodynamic interactions between Brownian particles near a surface. The findings of Dufresne et al. are particularly relevant to dynamical studies of polymers in confined geometries (microfluidic devices), where the velocity field generated by motion of the macromolecule is generally taken to be due to point forces acting on the interaction sites, or "beads" that constitute the chain.

We now present a method for the numerical evaluation of the hydrodynamic interaction tensor for general geometries. The level of treatment of $\mathrm{HI}$ in a bounded domain considered here is similar to that employed by Dufresne et $a l .{ }^{42}$ in their work. Note however, that closed form solutions only exist for Oseen-Burgers hydrodynamics in a few special cases (i.e., point particles between infinite planes or in straight channels with constant cross section); for general geometries the problem must be solved numerically.

The Green's function for Stokes flow in an arbitrary device can be expressed as

$$
\boldsymbol{\Omega}=\boldsymbol{\Omega}^{\mathrm{OB}}+\boldsymbol{\Omega}^{W},
$$

where $\boldsymbol{\Omega}^{\mathrm{OB}}$ is the free-space Green's function [Eq. (4)] and $\boldsymbol{\Omega}^{W}$ is a correction which accounts for the no-slip constraint on the walls. The velocity perturbation due to a point force acting at $\mathbf{x}_{j}$ is then given as

$$
\begin{aligned}
\mathbf{v}^{\prime}\left(\mathbf{x}, \mathbf{x}_{j}\right) & =\mathbf{v}_{\mathrm{OB}}^{\prime}\left(\mathbf{x}-\mathbf{x}_{j}\right)+\mathbf{v}_{\mathrm{W}}^{\prime}\left(\mathbf{x}, \mathbf{x}_{j}\right) \\
& =\left[\mathbf{\Omega}^{\mathrm{OB}}\left(\mathbf{x}-\mathbf{x}_{j}\right)+\boldsymbol{\Omega}^{W}\left(\mathbf{x}, \mathbf{x}_{j}\right)\right] \cdot \mathbf{f}\left(\mathbf{x}_{j}\right) .
\end{aligned}
$$

Thus, $\mathbf{v}_{W}^{\prime}\left(\mathbf{x}, \mathbf{x}_{j}\right)$ can be obtained as the solution to the incompressible Stokes flow problem

$$
-\nabla p+\eta_{s} \nabla^{2} \mathbf{v}_{W}^{\prime}=0, \quad \nabla \cdot \mathbf{v}_{W}^{\prime}=0,
$$

subject to

$$
\mathbf{v}_{\mathrm{OB}}^{\prime}+\mathbf{v}_{W}^{\prime}=0
$$

at the walls. The wall Green's function, $\boldsymbol{\Omega}^{W}\left(\mathbf{x}, \mathbf{x}_{j}\right)$, can be obtained by the following procedure. First, one takes $\mathbf{v}_{\mathrm{OB}}^{\prime}(\mathbf{x}$ $\left.-\mathbf{x}_{j}\right)$ to be due to a point force $\left(f_{1}\right)$ acting in the $x_{1}$ direction, located at the point $\mathbf{x}_{j}$. The Stokes flow problem can be solved numerically to obtain $\mathbf{v}_{W}^{\prime}\left(\mathbf{x}, \mathbf{x}_{j}\right)$. This gives the first column of $\boldsymbol{\Omega}^{W}\left(\mathbf{x}, \mathbf{x}_{j}\right)$ by

$$
\left(\begin{array}{l}
\Omega_{11}^{W} \\
\Omega_{21}^{W} \\
\Omega_{31}^{W}
\end{array}\right)=\frac{1}{f_{1}} \mathbf{v}_{W}^{\prime} .
$$

Similarly, the second and third columns of $\boldsymbol{\Omega}^{W}\left(\mathbf{x}, \mathbf{x}_{j}\right)$ are obtained by applying point forces in the $x_{2}$ and $x_{3}$ directions, respectively. Now, $\boldsymbol{\Omega}_{i j}$, in Eq. (3) is given by

$$
\boldsymbol{\Omega}_{i j}=\boldsymbol{\Omega}^{W}\left(\mathbf{r}_{i}, \mathbf{r}_{j}\right)+\left(1-\delta_{i j}\right) \boldsymbol{\Omega}^{\mathrm{OB}}\left(\mathbf{r}_{i}-\mathbf{r}_{j}\right) .
$$

In contrast to the case of unbounded flow, here $\boldsymbol{\Omega}_{i j} \neq 0$ for $i=j$. Furthermore, $\partial / \partial \mathbf{r}_{i} \cdot \boldsymbol{\Omega}_{i j}^{W}$ is nonzero for $i=j$, resulting in a nonzero drift term in Eq. (1).

As in the bulk case (Sec. II), use of the Green's function in the above formulation leads to nonpositive-semidefinite $\mathbf{D}$ for some chain configurations. However, a diffusion tensor constructed by the above-outlined method using $\boldsymbol{\Omega}^{\mathrm{RPY}}$ in place of $\boldsymbol{\Omega}^{\mathrm{OB}}$ results in a nonsymmetric $\mathbf{D}$; this violates the reciprocity relation

$$
\boldsymbol{\Omega}\left(\mathbf{r}_{i}, \mathbf{r}_{j}\right)=\boldsymbol{\Omega}^{T}\left(\mathbf{r}_{j}, \mathbf{r}_{i}\right),
$$

which follows from self-adjointness of the Stokes operator. ${ }^{29}$ This violation is analogous to that which would occur if RPY hydrodynamic interactions were used naively for spheres of unequal size. ${ }^{43-45}$ For that situation, Felderhof ${ }^{43}$ performed a multipole expansion of the free-solution hydrodynamic interaction tensor. To order $1 / r^{3}$, the resulting hydrodynamic interaction tensor is identical (for $\left|r_{i j}\right| \geqslant 2 a$ ) to the symmetrized RPY tensor, $\boldsymbol{\Omega}_{i j}^{\mathrm{MP}}=\left(\boldsymbol{\Omega}_{i j}^{\mathrm{RPY}}+\boldsymbol{\Omega}_{j i}^{\mathrm{RPY}}\right) / 2$. By analogy with this result, we obtain a symmetric, positive-semidefinite diffusion tensor as follows. First, $\boldsymbol{\Omega}_{i j}^{\mathrm{OB}}$ is replaced by $\boldsymbol{\Omega}_{i j}^{\mathrm{RPY}}$ in Eqs. (15) and (17), giving a wall correction $\hat{\boldsymbol{\Omega}}_{i j}^{W}$, which is not equal to $\left(\hat{\boldsymbol{\Omega}}_{j i}^{W}\right)^{T}$. Then, a symmetric, positivesemidefinite diffusion tensor is calculated according to

$$
\boldsymbol{\Omega}_{i j}^{W}=\frac{\hat{\boldsymbol{\Omega}}_{i j}^{W}+\left(\hat{\boldsymbol{\Omega}}_{j i}^{W}\right)^{T}}{2} .
$$

We note here that we account for hydrodynamic interactions at the point force level (i.e., Oseen-Burgers in a confined geometry); the RPY formulation is employed only to maintain positive-semidefiniteness of the diffusion tensor. While the use of Eq. (19) to obtain a symmetric $\mathbf{D}$ is not rigorous, it is essentially a near-field regularization, and introduces an error in the point force hydrodynamics of order $a / H \ll 1$. Our treatment of hydrodynamic interaction may be considered as an approximate Rotne-Prager-Yamakawa formulation for confined geometries. For the special case of particles above an infinite plane wall, our treatment of the hydrodynamic interaction can be worked out analytically. We have done this and verified that our method is indeed a well-behaved nearfield modification of the near-wall point-force solution. We have included a demonstration of this in Appendix A. 


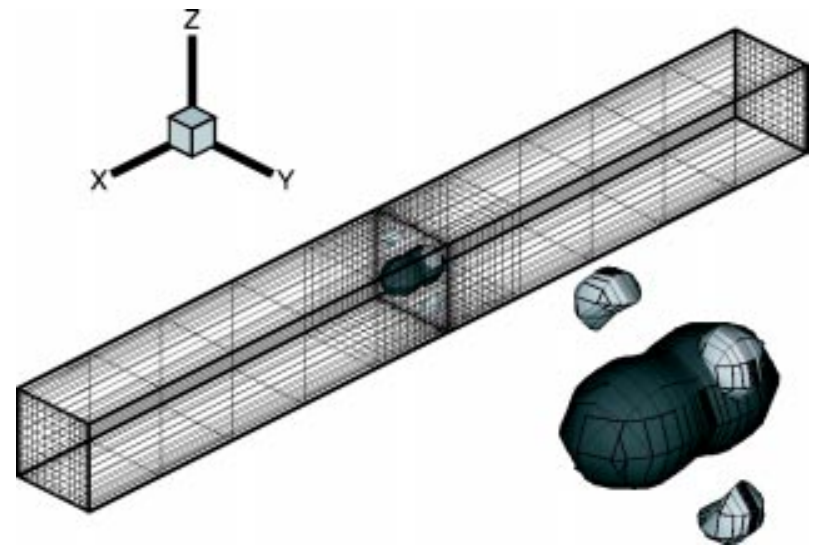

FIG. 1. Green's function meshing for a $63.6 \mu \mathrm{m} \times 6.36 \mu \mathrm{m} \times 6.36 \mu \mathrm{m}$ channel. Also shown are the $v_{x}=0.01 / \zeta$ (dark) and $-0.01 / \zeta$ (light) contours due to a point force in the $x$ direction of magnitude 1, located at the center of the channel. The inset is a close up view of the velocity contours.

The evaluation of the Green's function is performed once, in a preprocessing step, for a given device or geometry; $\boldsymbol{\Omega}^{W}\left(\mathbf{x}_{i}, \mathbf{x}_{j}\right)$ is obtained numerically on a grid using a finite element method. ${ }^{46}$ During a Brownian dynamics simulation, $\boldsymbol{\Omega}_{i j}^{W}$ and its divergence are obtained by finite element interpolation. ${ }^{47}$ Figure 1 shows a typical mesh used.

At first glance, it may appear that one needs $O\left(N_{G}^{2}\right)$ of the $\boldsymbol{\Omega}_{m n}^{W} \mathrm{~s}$, with $N_{G}$ being the number of grid points in the microfluidic domain. However, in confined geometries, the Green's function decays rapidly (exponentially, in a channel) ${ }^{40}$ and in practice one only needs to keep the $\boldsymbol{\Omega}_{m n}^{W} \mathrm{~s}$ for $\left|\mathbf{x}_{n}-\mathbf{x}_{m}\right|<x_{o}$, where $x_{o}$ is a cutoff distance which depends on the proximity of the point $\mathbf{x}_{j}$ to the device walls. Thus, in practical applications, one needs $O\left(N_{G}\right)$ of the $\boldsymbol{\Omega}_{m n}^{W} \mathrm{~s}$, with the coefficient of proportionality depending on the details of the computational domain.

For comparison to the detailed HI model described above, we will present some results for three approximate models. In the bulk hydrodynamic interaction model, we simply ignore walls in computing the hydrodynamics; $\boldsymbol{\Omega}^{W}$ is set to zero. In the reduced mobility (RM) model, hydrodynamic interactions between particles are neglected, while modifications to the bead mobility due to boundaries are maintained. In that case the diffusion tensor [Eq. (3)] becomes block diagonal

$$
\mathbf{D}_{i j}^{\mathrm{RM}}=k_{B} T \delta_{i j}\left(\frac{1}{6 \pi \eta a} \mathbf{I}+\boldsymbol{\Omega}_{i j}^{W}\right) .
$$

In the free-draining (FD) model, which has been favored in many literature studies of DNA, both hydrodynamic interactions and modifications to the bead mobility are neglected, resulting in a position-independent, isotropic diffusion tensor

$$
\mathbf{D}_{i j}^{\mathrm{FD}}=\delta_{i j} \frac{k_{B} T}{6 \pi \eta a} \mathbf{I}
$$

In this work, we consider the behavior of individual DNA molecules in an infinitely long microchannel with square cross section. The center line of the channel is oriented along the $x$ axis, with the cross section lying in the $y z$ plane. The imposed velocity field in the channel is given by ${ }^{48}$

$$
\begin{aligned}
\mathbf{v}(x, y)= & \frac{4 H^{2}}{\eta \pi^{3}}\left(-\frac{d p}{d x}\right) \sum_{k=1,3,5, \ldots}^{\infty}(-1)^{(k-1) / 2} \\
& \times\left[1-\frac{\cosh (k \pi z / H)}{\cosh (k \pi / 2)}\right] \frac{\cos (k \pi y / H)}{k^{3}},
\end{aligned}
$$

where $p$ is the pressure. The strength of the flow is defined through an effective shear rate, $\dot{\gamma}_{\text {eff }}=2 v_{\max } / H$, where $v_{\text {max }}$ $=|\mathbf{v}(y=0, z=0)|$ is the imposed velocity at the center line of the channel.

The physical confinement of the molecule is taken into account through a simple bead-wall repulsive potential of the form

$$
\begin{aligned}
U_{i}^{\text {wall }} & =\frac{A_{\text {wall }}}{3 b_{k} \delta_{\text {wall }}^{2}}\left(h-\delta_{\text {wall }}\right)^{3} \text { for } h<\delta_{\text {wall }} \\
& =0 \quad \text { for } h \geqslant \delta_{\text {wall }},
\end{aligned}
$$

where $h$ represents the distance of bead $i$ from the wall in the wall-normal direction (into the fluid). Throughout this work, we take $A_{\text {wall }}=25 k_{B} T$ and $\delta_{\text {wall }}=b_{k} N_{k, s}^{1 / 2} / 2=0.236 \mu \mathrm{m}$.

\section{Simulation}

In the limit of infinite channel length, a point force acting on the fluid generates no net flow through the channel (i.e., in the $x$ direction), even if the channel ends are open; for there to be a net flow the force would need to be infinite. For the numerical evaluation of the hydrodynamic interaction tensor, the infinitely long channel is therefore approximated by a closed channel of length $10 \mathrm{H}$, at which point the results are insensitive to further increase in length. Figure 1 shows the mesh and $v_{x}=0.01 / \zeta$ (dark) and $-0.01 / \zeta$ (light) contour surfaces for a unit point force in the $x$ direction, located at the center of a $6.3 \mu \mathrm{m}$ wide channel.

Equation (1) was evolved in time using a semi-implicit integration scheme described in our previous papers. ${ }^{26,27}$ The range of the hydrodynamic interaction is proportional to the channel width $H$; all components of $\mathbf{D}$ less than $0.001 \max \left(D_{i i}\right)$ were neglected. The decomposition of $\mathbf{D}$ [Eq. (2)] is computationally demanding, and was achieved using a fast algorithm proposed by Fixman ${ }^{30}$ and implemented as described in our earlier work. ${ }^{26}$ For general robustness, we also implemented the method outlined in Appendix B to ensure that $\mathbf{D}$ remained positive semidefinite. The simulations were performed in dimensionless variables, with lengths, times and energies scaled by $b_{k}, 6 \pi \eta b_{k}^{2} / k_{B} T$ and $k_{B} T$, respectively. A time step of $\Delta t=0.1 \min \left[\left(6 \pi \eta a S_{s}^{2}\right) /\right.$ $\left.\left(k_{B} T\right), \dot{\gamma}_{\text {eff }}^{-1}\right]$ was used in all simulations. All results are presented for DNA at room temperature in a $1 \mathrm{cP}$ solvent. Chains ranging from $10.5 \mu \mathrm{m}\left(N_{s}=5\right)$ to $126 \mu \mathrm{m}\left(N_{s}\right.$ $=60$ ) were simulated in channels having widths in the range of $0.636-10.6 \mu \mathrm{m}$. For each molecular weight, results were collected for 20 trajectories over a period of 2000 bulk relaxation times based on a $42 \mu \mathrm{m}$ chain, about $650 \mathrm{~s}$. Note that the level of molecular discretization was kept constant at $N_{k, s}=19.8$. We investigated the effect of molecular discretization on the full model by using $N_{k, s}=13.2$ in simulations of 42,126 , and $210 \mu \mathrm{m}$ chains in $1.6 \mu \mathrm{m}$ channels; the results were within a few percent of the coarser representa- 
TABLE I. Equilibrium bulk radius of gyration, bulk relaxation time, and bulk Weissenberg number at various shear rates, from simulations for different chain contour lengths.

\begin{tabular}{cccccc}
\hline \hline$L(\mu \mathrm{m})$ & $S_{b}(\mu \mathrm{m})$ & $\lambda_{1, b}(\mathrm{~s})$ & We @ $\dot{\gamma}_{\mathrm{eff}}=3.98 \mathrm{~s}^{-1}$ & We @ $\dot{\gamma}_{\mathrm{eff}}=30.8 \mathrm{~s}^{-1}$ & We @ $\dot{\gamma}_{\mathrm{eff}}=308 \mathrm{~s}^{-1}$ \\
\hline 10.5 & 0.52 & 0.03 & 0.12 & 0.92 & 9.2 \\
21 & 0.76 & 0.09 & 0.36 & 2.8 & 28 \\
42 & 1.14 & 0.3 & 1.2 & 9.2 & 92 \\
84 & 1.73 & 0.9 & 3.6 & 28 & 280 \\
126 & 2.19 & 1.9 & 7.6 & 59 & 590 \\
\hline \hline
\end{tabular}

tion, lending credence to the predictive capabilities of the model. The results were also found to be fairly insensitive to the wall parameter $\delta$, provided $\delta=O(a)$.

\section{Properties}

The following properties are considered in this work. The instantaneous "stretch" of the chain $\bar{X}$ is defined as the absolute length of the molecule in the channel direction

$$
\bar{X}=\max _{i}\left(r_{i, x}\right)-\min _{i}\left(r_{i, x}\right),
$$

where $r_{i, x}$ is the $x$ component of the position vector of bead $i$. The steady-state stretch of the molecule is defined as

$$
X=\langle\bar{X}\rangle_{N},
$$

where

$$
\langle A\rangle_{N}=\frac{1}{N} \sum_{n=1}^{N} A_{n}
$$

represents a steady-state (ensemble) average over $N$ observations and $A_{n}$ is the value of $A$ at observation $n$.

The longest relaxation time of a chain, $\lambda_{1}$, is calculated by allowing a chain that is initially fully stretched along the center line of the channel to relax to equilibrium. Near equilibrium, the relaxation time is determined by a fit to an exponential decay

$$
\left\langle\bar{X}^{2}\right\rangle_{N}(t)=\left(\bar{X}^{2}(0)-\left\langle\bar{X}^{2}\right\rangle_{N, \text { eq }}\right) \exp \left(-\frac{t}{\lambda_{1}}\right)+\left\langle\bar{X}^{2}\right\rangle_{N, \text { eq }},
$$

where \langle\rangle$_{N \text {, eq }}$ indicates an equilibrium average. The radius of gyration of the chain $S$ is defined through

$$
S^{2}=\left\langle\frac{1}{N_{b}} \sum_{i=1}^{N_{b}}\left|\mathbf{r}_{i}-\mathbf{r}_{c}\right|^{2}\right\rangle_{N},
$$

where $\mathbf{r}_{c}$ is the center of mass of the molecule. We also define $X_{b}, \lambda_{1, b}$, and $S_{b}$ as the equilibrium bulk values of the stretch, longest relaxation time, and radius of gyration, respectively. Table I gives the simulation predictions for the bulk equilibrium radius of gyration and relaxation time for different chain contour lengths. It also gives the nominal (bulk) Weissenberg number $\mathrm{We}=\lambda_{1, b} \dot{\gamma}_{\text {eff }}$ for each molecular weight at the three shear rates considered in this paper.

The steady state center of mass distribution in the cross section of the channel is given by

$$
P_{c}(y, z)=\left\langle\delta\left(y-y_{c, n}\right) \delta\left(z-z_{c, n}\right)\right\rangle_{N},
$$

where $y_{c, n}$ and $z_{c, n}$ are the $y$ and $z$ coordinates of the center of mass of the molecule at observation $n$. The second moment of the center of mass distribution in the cross section of the channel is

$$
w^{2}=\int\left(y^{2}+z^{2}\right) P_{c}(y, z) d y d z .
$$

The quantity $w / w_{\text {eq }}$ gives a measure of the width of the center of mass distribution relative to the equilibrium value. The intramolecular mass distribution in the axial direction is given by

$$
g\left(x-x_{c}\right)=\left\langle\frac{1}{N_{b}} \sum_{i=1}^{N_{b}} \delta\left(\left|x-x_{c}\right|-\left|x_{i, n}-x_{c, n}\right|\right)\right\rangle_{N},
$$

where $x_{i, n}$ and $x_{c, n}$ are the axial positions of bead $i$ and the chain center of mass at observation $n$.

\section{RESULTS}

\section{A. Onset of flow-induced stretching}

We first present results on the steady-state stretch of DNA chains in pressure-driven flow through square microchannels. Figure 2 shows the steady-state stretch as a function of channel width for various chain lengths and flow strengths. Consider, for example, the results for a $126 \mu \mathrm{m}$ chain at a flow strength of $\dot{\gamma}_{\text {eff }}=30.8 \mathrm{~s}^{-1}, \mathrm{We}=59$ [triangles

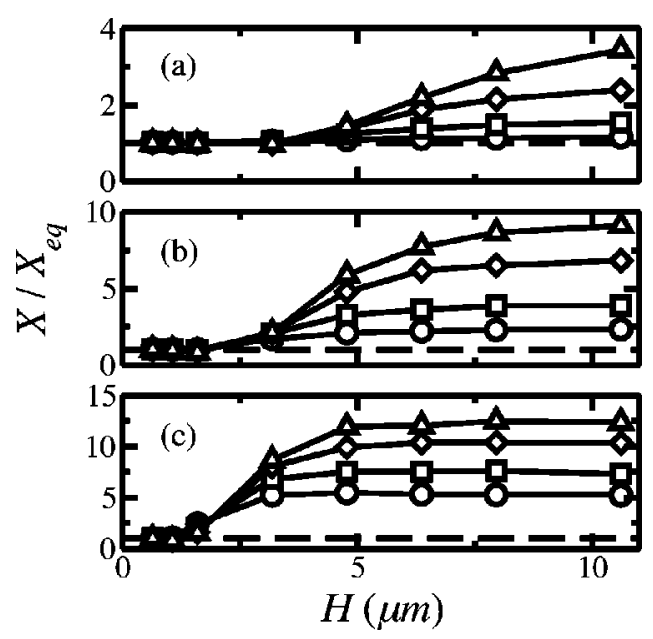

FIG. 2. Steady-state stretch reduced by the equilibrium stretch as a function of channel width: (a) $\dot{\gamma}_{\text {eff }}=3.98 \mathrm{~s}^{-1}$, (b) $\dot{\gamma}_{\text {eff }}=30.8 \mathrm{~s}^{-1}$, (c) $\dot{\gamma}_{\text {eff }}=308 \mathrm{~s}^{-1}$. Symbols refer to chains lengths of $21 \mu \mathrm{m}(\bigcirc), 42 \mu \mathrm{m}(\square), 84 \mu \mathrm{m}(\diamond)$, and $126 \mu \mathrm{m}(\triangle)$. These chain lengths have equilibrium free-solution radii of gyration of $0.76,1.14,1.73$, and $2.19 \mu \mathrm{m}$, respectively. 
in Fig. 2(b)]. At small $H$, the chain is elongated or cigarshaped at equilibrium. The first of effect the flow is to compress the chain slightly in the axial direction and push the "cigar" along the channel (compression of the chain will be demonstrated more clearly below). As the level of confinement decreases, a channel size $\left(H_{c}\right)$ is eventually reached where the flow strength is sufficient to cause the chain to stretch away from its equilibrium value. Figures 2(a) -2 (c) indicate that the value of $H$ at which the chain stretches away from its equilibrium value (for that channel size) is independent of molecular weight and decreases with increasing flow strength. For example, in Fig. 2(a) $\left(\dot{\gamma}_{\text {eff }}=3.98 \mathrm{~s}^{-1}\right)$ all chain lengths (symbols) stretch at the same value of $H$. This is also true for the stronger flows [Figs. 2(b) and 2(c)], although the value of $H_{c}$ decreases. Using a simple "blob" model, ${ }^{49-51}$ one expects that, in homogeneous simple shear with shear rate $\dot{\gamma}$, a highly confined chain will begin to stretch away from its equilibrium value when the blob Weissenberg number $\mathrm{We}_{\text {blob }}=\dot{\gamma} \lambda_{\text {blob }}$ is order unity, where $\lambda_{\text {blob }}$ is the blob longest relaxation time, i.e., the relaxation time of a coil with size $\approx H$. In a good solvent, $H=b_{k} N_{\text {blob }}^{\nu}$, where $N_{\text {blob }}^{\nu}$ is the number of Kuhn segments per blob, and the exponent $\nu$ $\approx 3 / 5$. The Zimm longest relaxation time of such a blob is approximated by ${ }^{52} \quad \lambda_{\text {blob }} \approx\left(\eta_{s} b_{k}^{3}\right) /\left(k_{B} T\right) N_{\text {blob }}^{3 \nu}$ $=\left(\eta_{s} H^{3}\right) /\left(k_{B} T\right)$. Note that the stiffness of the chain does not appear explicitly in this expression for the relaxation time of a blob; this is for $H \gg b_{k}$. The Weissenberg number based on the blob good solvent Zimm relaxation time is $\mathrm{We}_{\text {blob }}=\lambda_{\text {blob }} \dot{\gamma} \approx\left(\eta_{s} H^{3} \dot{\gamma}\right) /\left(k_{B} T\right)$, and the chain is expected to stretch when

$$
H^{3}>H_{c}^{3} \sim \frac{k_{B} T}{\eta_{s} \dot{\gamma}} .
$$

Equation (33) gives two interesting results. First, the critical shear rate is independent of molecular weight; our simulations (Fig. 2) agree with this; for a given $\dot{\gamma}_{\text {eff }}$, all chain lengths stretch away from the equilibrium value at the same value of $H$. Second, Eq. (33) predicts $H_{c} \propto\left(\dot{\gamma}_{\text {eff }}\right)^{-1 / 3}$. Although our simulations show a definite decrease in $H_{c}$ with increasing $\dot{\gamma}_{\text {eff }}$, the relation between the two does not appear to satisfy the power law relation in Eq. (33). This discrepancy is not surprising since the scaling argument assumed simple shear, while we have a nonuniform velocity gradient. Finally, we note that for the longest chain at the highest flow rate, there is a slight dip in the curve of $X / X_{\text {eq }}$ for $H$ just below the onset of stretching [triangles on Fig. 2(c)]. For this confined chain $\left(H / S_{b} \approx 0.5\right)$ at this high flow rate, the chain is axially compressed a small amount as it moves down the channel.

\section{B. Cross-sectional distributions: Cross-stream migration and its consequences}

The effect of flow on the chain center of mass distribution in the cross section of the channel is illustrated in Fig. 3 for a $42 \mu \mathrm{m}$ chain in a $10.6 \mu \mathrm{m}$ channel $\left(H / S_{b} \approx 9.3-\mathrm{a}\right.$ weakly confined chain). At equilibrium, the distribution is fairly uniform across the channel, with a static depletion layer near the walls whose size is roughly $S_{b}(1.14 \mu \mathrm{m})$. As
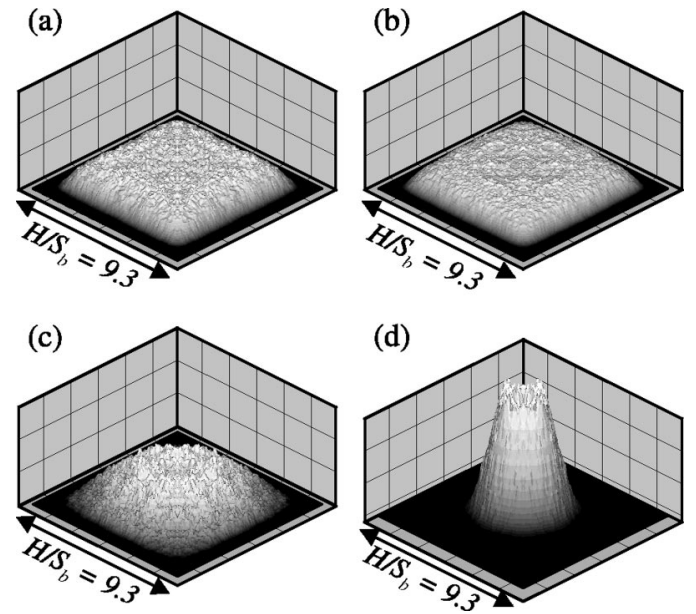

FIG. 3. Steady-state center-of-mass distribution $P_{c}(y, z)$ in the cross section of the channel for a $42 \mu \mathrm{m}$ chain in a $10.6 \mu \mathrm{m}$ wide channel: (a) $\dot{\gamma}_{\text {eff }}$ $=0 \mathrm{~s}^{-1} \quad(\mathrm{We}=0)$, (b) $\dot{\gamma}_{\mathrm{eff}}=3.98 \mathrm{~s}^{-1} \quad(\mathrm{We}=1.2)$, (c) $\dot{\gamma}_{\mathrm{eff}}=30.8 \mathrm{~s}^{-1}$ (We $=9.2)$, (d) $\dot{\gamma}_{\text {eff }}=308 \mathrm{~s}^{-1}(\mathrm{We}=92)$. Grid line spacing is $2 \mu \mathrm{m}$ in the $y z$ plane and 0.2 for $P_{c}(y, z)$

flow strength increases, a hydrodynamically induced depletion layer (dark areas) forms near the walls-this observation is discussed in detail below. The effect of flow on the chain stretch distribution in the cross section of the channel is illustrated in Fig. 4 for a $42 \mu \mathrm{m}$ chain in a $10.6 \mu \mathrm{m}$ channel. These plots represent the conditional average stretch-the average stretch for chains found at that particular position in the channel. The results are plotted this way to decouple the chain stretching at a particular position from the probability of finding a chain at that position, which is nonuniform as shown in Fig. 3. The black areas in Fig. 4 represent regions where no chains were observed at large times. At equilibrium, the chain stretch is fairly uniform across the channel. Chains within the static depletion layer are more highly
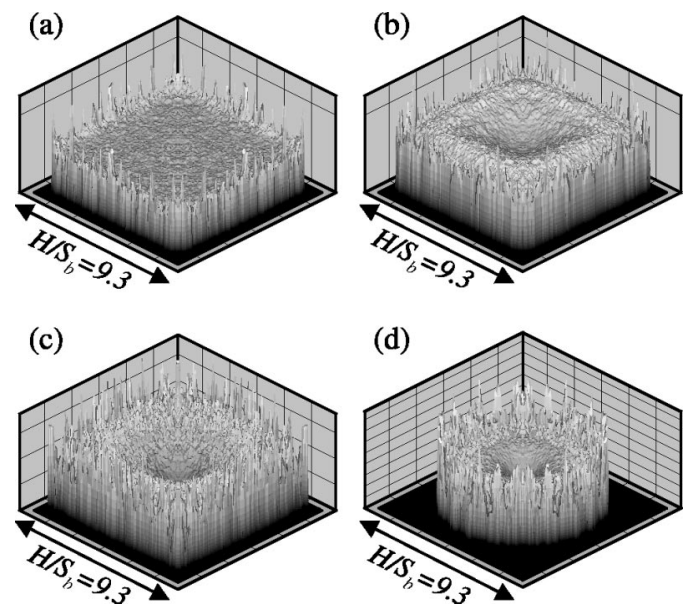

FIG. 4. Steady-state stretch $X(y, z)$ as a function of location of chain center of mass in the cross section of the channel. Results are for a $42 \mu \mathrm{m}$ chain in $10.6 \mu \mathrm{m}$ wide channel: (a) $\dot{\gamma}_{\mathrm{eff}}=0 \mathrm{~s}^{-1}(\mathrm{We}=0)$, (b) $\dot{\gamma}_{\mathrm{eff}}=3.98 \mathrm{~s}^{-1}$ (We $=1.2)$, (c) $\dot{\gamma}_{\mathrm{eff}}=30.8 \mathrm{~s}^{-1}(\mathrm{We}=9.2)$, (d) $\dot{\gamma}_{\mathrm{eff}}=308 \mathrm{~s}^{-1}(\mathrm{We}=92)$. Grid line spacing is $2 \mu \mathrm{m}$ in the $y z$ plane and $4 \mu \mathrm{m}$ for $X(y, z)$. Values of zero (black) represent regions where chains were not observed (see Fig. 3 and corresponding discussion). The data around the edges are noisy due the small number of observations (small probability of finding a chain there). 

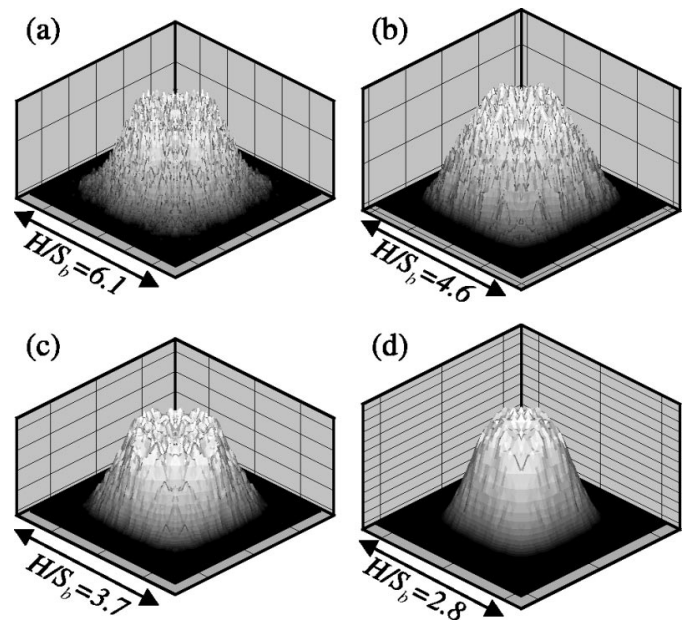

FIG. 5. Steady-state center-of-mass distribution $P_{c}(y, z)$ in the cross section of the channel for an $84 \mu \mathrm{m}$ chain at a flow strength of $\dot{\gamma}_{\text {eff }}=30.8 \mathrm{~s}^{-1}$ (We $=28$ ) in channel widths of: (a) $10.6 \mu \mathrm{m}$, (b) $7.95 \mu \mathrm{m}$, (c) $6.36 \mu \mathrm{m}$, and (d) $4.77 \mu \mathrm{m}$. Grid line spacing is $2 \mu \mathrm{m}$ in the $y z$ plane and 0.2 for $P_{c}(y, z)$.

stretched due to steric constraints-only a highly elongated molecule can fit near the wall. At higher flow strengths the distribution becomes highly nonuniform. Near the center line, chains are not stretched, as the shear rate is small there. Moving toward the wall the stretch increases, but at least at intermediate Weissenberg numbers [Fig. 4(b)], the stretch seems to peak about halfway between the wall and center line, then decreases again as the wall is approached, even though the strain rate is increasing. Finally, very close to the wall (around one radius of gyration away), the stretch becomes large again. An unexpectedly low degree of polymer stretch near a wall during shear flow of DNA has recently been experimentally observed. ${ }^{53}$ The origin of this unexpected behavior is as yet unknown, but it may be that the more highly stretched chains migrate to the center faster than less stretched ones, so the latter are more likely to be found near the wall than would normally be expected. This argument is supported by a comparison between the stretch and center-of-mass distributions, which indicates that although chains tend to be highly stretched where the local velocity gradient is higher, it is much less likely to find a chain in that region. It is also consistent with the theoretical development described in Sec. IV. Figure 5 shows the chain center-ofmass distribution for a longer chain $(84 \mu \mathrm{m})$ at a flow strength of $\dot{\gamma}_{\text {eff }}=30.8 \mathrm{~s}^{-1}$ in various channel sizes.

The nonequilibrium, steady-state center-of-mass distributions in Figs. 3 and 5 indicate a nonmonotonic concentration profile in the cross section of the channel. We remind the reader that these are dilute-solution results (single-molecule simulations) so "concentration" is really "center-of-mass probability density." There is a shallow local minimum at the center line, a maximum off the center line, then a monotonically decreasing concentration toward the walls, with substantial depletion of polymer near the walls. This nonmonotonic concentration profile is due to a competition of Brownian migration away from the center line (due to coupling of hydrodynamics with spatial variations in the polymer conformational distribution function) and deterministic

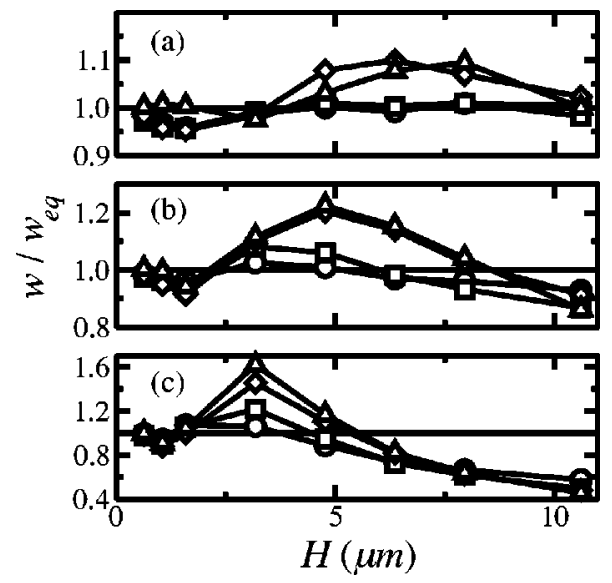

FIG. 6. Width of the steady-state center-of-mass distribution (relative to equilibrium) as a function of channel width for chain lengths of $21 \mu \mathrm{m}(\bigcirc)$, $42 \mu \mathrm{m}(\square), 84 \mu \mathrm{m}(\diamond)$, and $126 \mu \mathrm{m}(\triangle)$ : (a) $\dot{\gamma}_{\text {eff }}=3.98 \mathrm{~s}^{-1}$, (b) $\dot{\gamma}_{\text {eff }}$ $=30.8 \mathrm{~s} \mathrm{~s}^{-1}$, (c) $\dot{\gamma}_{\mathrm{eff}}=308 \mathrm{~s}^{-1}$. The equilibrium free-solution radius of gyration of the $21,42,84$, and $126 \mu \mathrm{m}$ chains is $0.76,1.14,1.73$, and $2.19 \mu \mathrm{m}$, respectively. Note the different scales on the vertical axis.

migration away from the walls (due to asymmetry in the wall hydrodynamic interaction). These mechanisms will be discussed in detail in Sec. IV.

One way of quantifying the depletion effect is to consider the width $w$ [Eq. (31)], of the center-of-mass distribution. Figure 6 shows $w$, reduced by the equilibrium value $w_{\text {eq }}$, as a function of channel width for various molecular weight chains and flow strengths. Using as an example the diamond symbols in Fig. 6(b) $(84 \mu \mathrm{m}$ chain, flow strength $\dot{\gamma}_{\text {eff }}=30.8 \mathrm{~s}^{-1}$ ), we identify three regions of interest. As mentioned above, for small $H / S_{b}$ one of the first effects of the flow is to compress the chain slightly in the axial direction. This effective increase in monomer density reduces the transverse fluctuations of the chain center of mass resulting in $w / w_{\text {eq }}<1$. As channel width increases, the flow is eventually able to stretch the chain, allowing the chain center of mass to sample closer to the walls, which results in $w / w_{\text {eq }}$ $>1$. As channel width is increased further, hydrodynamic depletion effects become important and $w / w_{\text {eq }}$ again decreases below unity.

At all flow strengths considered here, $w$ decreases with increasing molecular weight, indicating that a higher molecular weight chain will on average spend more time in regions of higher axial fluid velocity than a lower molecular weight chain. This leads one to imagine that a microchannel could be used as a separation device. Figure 7 illustrates this capability for a flow strength of $\dot{\gamma}_{\text {eff }}=308 \mathrm{~s}^{-1}$ in a $10.6 \mu \mathrm{m}$ channel, showing the axial distance traveled by a chain center of mass as a function of distance traveled by a fluid element at the center of the channel $\left(v_{\max } t\right)$. Results are shown for 20 trajectories at each of several molecular weights. The fact that distinct "bands" can be observed for different length DNA chains indicates that this phenomenon can be used to separate DNA molecules in small channels. We have also performed dilute multiple-chain simulations using a $10.6 \mu \mathrm{m}$ square channel of (periodic) length $84 \mu \mathrm{m}$ containing four $42 \mu \mathrm{m}$ chains and eight $21 \mu \mathrm{m}$ chains at a flow strength of $\dot{\gamma}_{\text {eff }}=308 \mathrm{~s}^{-1}$. This corresponds to a volume frac- 


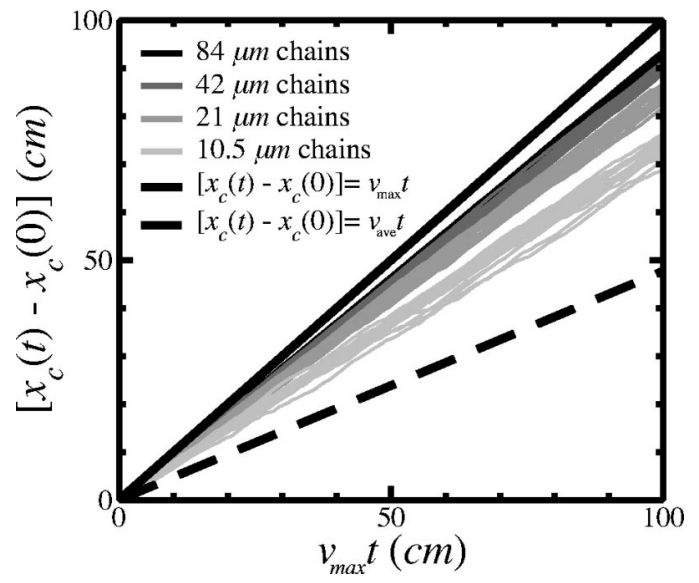

FIG. 7. Axial distance traveled by individual chains vs axial distance traveled by a fluid element at the center line of the channel. Results are for a $10.6 \mu \mathrm{m}$ channel at a flow strength of $\dot{\gamma}_{\text {eff }}=308 \mathrm{~s}^{-1}$. From darkest to lightest, shades correspond to $84,42,21$, and $10.5 \mu \mathrm{m}$ chains, respectively. Twenty trajectories from each molecular weight are shown. The solid and dashed thick lines are the results one would obtain if a chain moved at the maximum or average fluid velocity, respectively.

tion of 0.004 based on the free-solution radius of gyration of the DNA chains. The results of these simulations were identical to the results in Fig. 7; dilute chain-chain interactions do not appear to have a noticeable impact on the average velocity of the chains. Note that in one of these dilute multiple-chain simulations, a $42 \mu \mathrm{m}$ chain will pass by a 21 $\mu \mathrm{m}$ chain $\approx 600$ times in generating results such as those in Fig. 7.

The slope of the results in Fig. 7 gives $v_{c}$, the average chain velocity in the axial direction. Figure 8 shows the average chain velocity (reduced by $v_{\max }$ ) as a function of channel size for various chain lengths and flow strengths-these results correspond to those for $w$ given in Fig. 6. Note the nonmonotonic behavior of $v_{c} / v_{\max }$ for the $\dot{\gamma}_{\mathrm{eff}}=308 \mathrm{~s}^{-1}$ flow. The data in Fig. 8 collapse at small $H$ because the chains are highly confined and have not yet stretched away from their equilibrium configuration. These highly confined

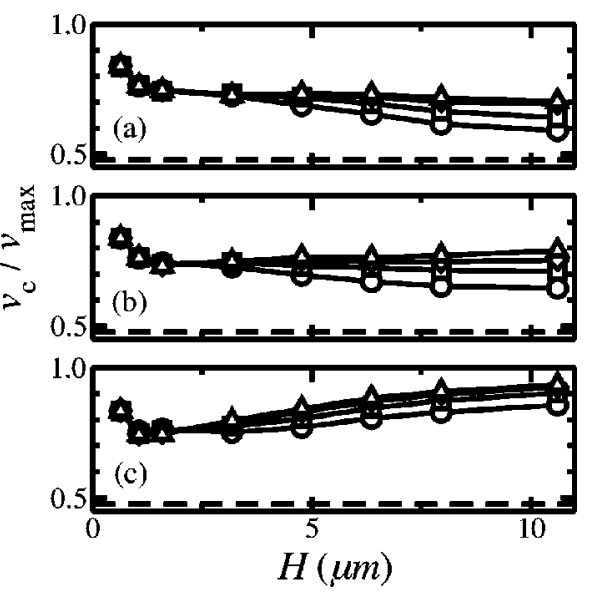

FIG. 8. Average chain axial velocity (reduced by the maximum fluid velocity) as a function of channel size: (a) $\dot{\gamma}_{\text {eff }}=3.98 \mathrm{~s}^{-1}$, (b) $\dot{\gamma}_{\mathrm{eff}}=30.8 \mathrm{~s}^{-1}$, and (c) $\dot{\gamma}_{\text {eff }}=308 \mathrm{~s}^{-1}$. Symbol types correspond to chain lengths of $21 \mu \mathrm{m}(\bigcirc)$, $42 \mu \mathrm{m}(\square), 84 \mu \mathrm{m}(\diamond)$, and $126 \mu \mathrm{m}(\triangle)$. The dashed line represents the average imposed axial fluid velocity.
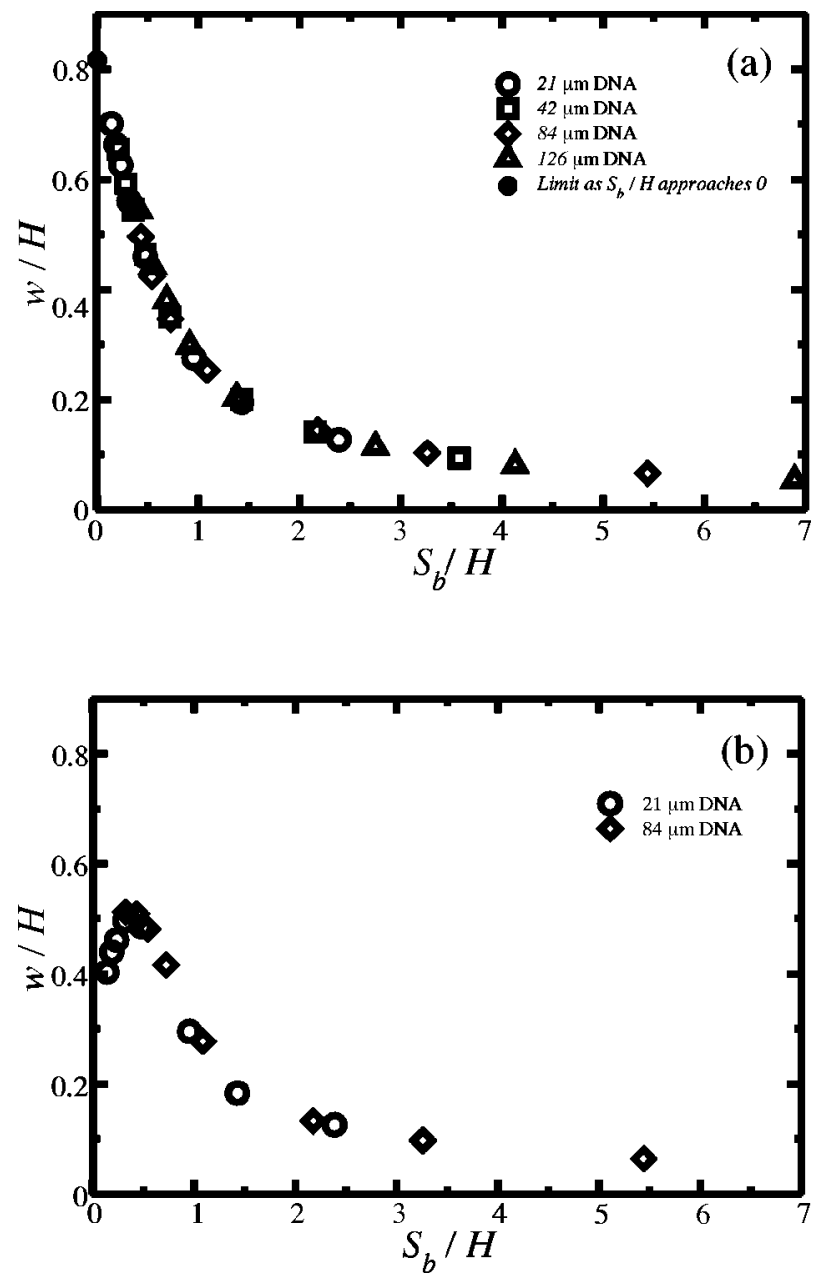

FIG. 9. Scaled width of the center-of-mass distribution $w / H$ vs $S_{b} / H$ for: (a) $\mathrm{We}=0$, (b) $\mathrm{We}=28$.

chains move at a velocity greater than the average imposed fluid velocity because they are excluded from regions near the walls. After the initial decrease for small $H, v_{c} / v_{\max }$ begins to increase due to hydrodynamic depletion effects.

Figure 9 shows some of the data of Fig. 6, replotted in dimensionless form, at equilibrium [Fig. 9(a)] and at We $=28$ [Fig. 9(b)]. The results collapse well onto master curves, lending confidence to our coarse graining. For $S_{b} / H \geqslant 1$, the width of the distribution is unchanged from the equilibrium value, indicating that the shear-induced depletion effect arises predominantly for weakly confined chains, $S_{b} / H>1$. For sufficiently large channels $\left(S_{b} / H \rightarrow 0\right)$, we expect that the hydrodynamic wall effects that lead to the depletion layer (see below) will become negligible on the scale of the channel, and that $w / H$ will attain its value for a uniform mass distribution $(\sqrt{2 / 3})$ independent of We. Whether this will be the case is unclear from the present results; resolution of this issue is the subject of future work.

As mentioned in Sec. II, it is well known that deformable particles in this type of flow experience a hydrodynamic driving force toward the center line of the channel. ${ }^{10,22}$ To clarify the origin of the driving force for our case of long flexible chains, we performed non-Brownian simulations by "turning off" the Brownian contributions (the $\partial / \partial \mathbf{r} \cdot \mathbf{D}$ and 


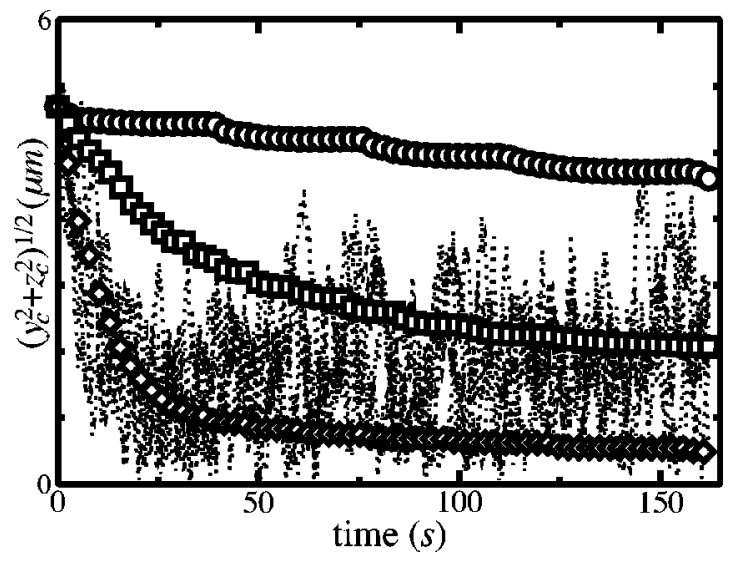

FIG. 10. Distance of center of mass position from the channel centerline $\left(\sqrt{y_{c}^{2}+z_{c}^{2}}\right)$ vs time for $42 \mu \mathrm{m}$ non-Brownian and Brownian chains in pressure-driven flow through a $10.6 \mu \mathrm{m}$ channel $\left(H / S_{b} \approx 9.3\right)$. All trajectories were started from the same equilibrium configuration with center of mass located at $\left(y_{c}, z_{c}\right)=(-1.9,-4.5) \mu \mathrm{m}$. Symbols correspond to $\dot{\gamma}_{\text {eff }}$ $=3.08 \mathrm{~s}^{-1}(\bigcirc), \dot{\gamma}_{\mathrm{eff}}=30.8 \mathrm{~s}^{-1}(\square)$, and $\dot{\gamma}_{\mathrm{eff}}=308 \mathrm{~s}^{-1}(\diamond)$. The dotted lines represent several Brownian trajectories for $\dot{\gamma}_{\mathrm{eff}}=308 \mathrm{~s}^{-1} \quad(\mathrm{We}=92)$, all started from the same initial configuration.

B. $d \mathbf{w}$ terms) in Eq. (1), for a $42 \mu \mathrm{m}$ chain in a $10.6 \mu \mathrm{m}$ channel $\left(H / S_{b}=9.3\right)$ at various flow strengths, with the chain initially located away from the center line of the channel. Figure 10 shows the distance of the chain center of mass from the center line, $\sqrt{y_{c}+z_{c}}$, as a function of time. There is clearly a deterministic hydrodynamic driving force toward the center line of the channel, and this force increases with increasing flow strength. Migration occurs while the chain is flipping-this is apparent from the "steps" in the $30.8 \mathrm{~s}^{-1}$ results (square symbols in Fig. 10). The thin lines in Fig. 10 represent Brownian trajectories for a flow strength of $\dot{\gamma}_{\text {eff }}$ $=308 \mathrm{~s}^{-1}$ (the same flow strength as for the diamond symbols). For Brownian chains, the width of the steady-state center of mass distribution is ultimately determined by a balance between hydrodynamic drift and chain diffusion. Note that the time required to reach steady state in Fig. 10 is more than an order of magnitude longer than the relaxation time of the chain. As mentioned in Sec. I, for Brownian chains there is an additional contribution to the hydrodynamic drift. We will discuss these depletion mechanisms in more detail in Sec. IV.

Further insight into the migration phenomenon can be achieved by comparison of the full model with the approximate hydrodynamic interaction models introduced at the end of Sec. II B. Figure 11 shows the steady-state center-of-mass distribution for a $42 \mu \mathrm{m}$ chain in a $10.6 \mu \mathrm{m}$ channel $\left(H / S_{b}\right.$ $=9.6$ ) at a flow strength of $\dot{\gamma}_{\text {eff }}=308 \mathrm{~s}^{-1}$. Results are shown for our full model [Fig. 11(a)] and the three approximate formulations: bulk hydrodynamic interaction model [Fig. 11(b)], reduced mobility model [Fig. 11(c)], and freedraining model [Fig. 11(d)]. The bulk hydrodynamic interaction model predicts a minimum in concentration at the centerline of the channel followed by a monotonically increasing concentration toward the walls (with the expected steric depletion at the walls); the direction of migration in the bulk hydrodynamic interaction model is opposite that of the full model, and the magnitude is significantly less. The re-
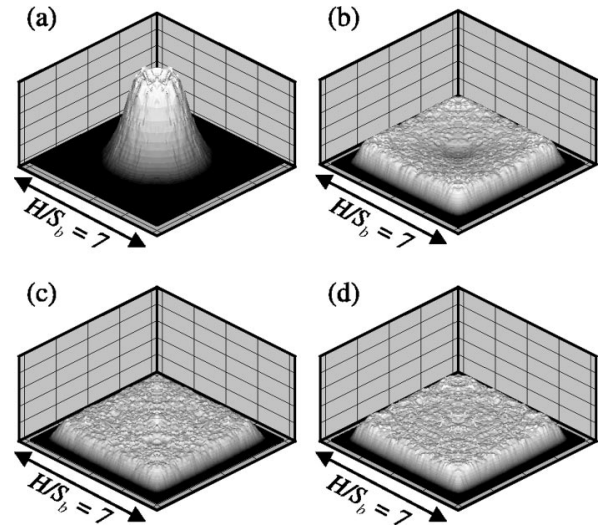

FIG. 11. Steady-state center-of-mass distribution $P_{c}(y, z)$ predicted by various models for $42 \mu \mathrm{m}$ chains in a $10.6 \mu \mathrm{m}$ channel at a flow strength of $\dot{\gamma}_{\text {eff }}=308 \mathrm{~s}^{-1}$ (We=92): (a) full hydrodynamic interactions, (b) bulk hydrodynamic interactions, (c) reduced mobility model, and (d) free-draining model. Grid line spacing is $2 \mu \mathrm{m}$ in the $y z$ plane and 0.2 for $P_{c}(y, z)$.

duced mobility model predicts a very slight increase in concentration at the center line, while the free-draining model predicts no migration at all. Note that all three of the approximate models show a slight narrowing of the steric depletion effect (relative to equilibrium) because the stretched chains can sample nearer the walls. ${ }^{21}$ These results indicate that the observed migration away from the walls is due to hydrodynamic interactions between confined chain segments. It turns out that all of the behaviors shown in Fig. 11 can be qualitatively explained using a simple kinetic theory dumbbell model of a confined polymer, as we now show.

\section{THEORY OF POLYMER MIGRATION IN RECTILINEAR FLOW: DUMBBELL MODEL}

\section{A. General framework}

In Sec. III we demonstrated that hydrodynamic interactions in a polymer solution flowing through channels of molecular dimension give rise to significant migration effects. We also showed in Fig. 11 that different approximations lead to qualitatively different cross-sectional concentration profiles. Here, we look at the migration effect within the context of the kinetic theory for a simple dumbbell - two beads connected by a spring - a representation of the confined polymer. The level of description is the same as we have been using in previous sections, except that excluded volume interactions are ignored (for simplicity). The configurational probability distribution function $(\Psi)$ for the polymer is a function of time $(t)$, the position of the center of mass $\left(\mathbf{r}_{c}\right.$ $\left.=\left[\mathbf{r}_{1}+\mathbf{r}_{2}\right] / 2\right)$ in physical space, and the connector vector $\left(\mathbf{q}=\mathbf{r}_{2}-\mathbf{r}_{1}\right)$. The governing equation for $\Psi\left(\mathbf{r}_{c}, \mathbf{q}, t\right)$ is ${ }^{7}$

$$
\frac{\partial \Psi}{\partial t}=-\frac{\partial}{\partial \mathbf{r}_{c}} \cdot\left(\dot{\mathbf{r}}_{c} \Psi\right)-\frac{\partial}{\partial \mathbf{q}} \cdot(\dot{\mathbf{q}} \Psi),
$$

where the momentum-space averaged velocity of the center of mass of the dumbbell, $\dot{\mathbf{r}}_{c}$, is given by ${ }^{7}$ 


$$
2 \dot{\mathbf{r}}_{c}=\sum_{i=1}^{2} \mathbf{v}_{i}+\frac{1}{k_{B} T} \sum_{i, j=1}^{2} \mathbf{D}_{i j} \cdot \mathbf{f}_{j}^{s}-\sum_{i, j=1}^{2} \mathbf{D}_{i j} \cdot \frac{\partial}{\partial \mathbf{r}_{j}} \ln \Psi,
$$

and the momentum-space averaged time rate of change of the connector vector, $\dot{\mathbf{q}}$, by $^{7}$

$$
\begin{aligned}
\dot{\mathbf{q}}= & {\left[\mathbf{v}_{2}-\mathbf{v}_{1}\right]+\frac{1}{k_{B} T} \sum_{j-1}^{2}\left(\mathbf{D}_{2 j}-\mathbf{D}_{1 j}\right) \cdot \mathbf{f}_{j}^{s}-\sum_{j-1}^{2}\left(\mathbf{D}_{2 j}-\mathbf{D}_{1 j}\right) } \\
& \cdot \frac{\partial}{\partial \mathbf{r}_{j}} \ln \Psi .
\end{aligned}
$$

Integrating Eq. (34) over $\mathbf{q}$ and defining

$$
\begin{aligned}
& \Psi\left(\mathbf{r}_{c}, \mathbf{q}, t\right)=C\left(\mathbf{r}_{c}, t\right) \hat{\Psi}\left(\mathbf{r}_{c}, \mathbf{q}, t\right), \\
& C\left(\mathbf{r}_{c}, t\right)=\int \Psi\left(\mathbf{r}_{c}, \mathbf{q}, t\right) d \mathbf{q},
\end{aligned}
$$

gives the governing equation for the center of mass probability distribution, $C\left(\mathbf{r}_{c}, t\right)$,

$$
\frac{\partial C}{\partial t}=-\frac{\partial}{\partial \mathbf{r}_{c}} \cdot \mathbf{j}_{c},
$$

where $\mathbf{j}_{c}=\left\langle\dot{\mathbf{r}}_{c}\right\rangle C$ is the momentum-space-averaged center-ofmass flux, integrated over the internal degrees of freedom of the molecule, and the angle brackets are defined through

$$
\langle A\rangle=\int A \hat{\Psi} d \mathbf{q} .
$$

Defining $\mathbf{f}^{s}=\mathbf{f}_{1}^{s}=-\mathbf{f}_{2}^{s}$ and using Eq. (3) along with

$$
\begin{gathered}
\frac{\partial}{\partial \mathbf{r}_{1}}=\frac{1}{2} \frac{\partial}{\partial \mathbf{r}_{c}}-\frac{\partial}{\partial \mathbf{q}}, \\
\frac{\partial}{\partial \mathbf{r}_{2}}=\frac{1}{2} \frac{\partial}{\partial \mathbf{r}_{c}}+\frac{\partial}{\partial \mathbf{q}},
\end{gathered}
$$

we can express Eq. (35) as

$$
\begin{aligned}
& \dot{\mathbf{r}}_{c}=\mathbf{v}_{c}+\frac{1}{2} \overline{\mathbf{\Omega}} \cdot \mathbf{f}^{s}+\frac{1}{2} \overline{\mathbf{D}} \cdot \frac{\partial}{\partial \mathbf{q}} \ln \Psi-\frac{1}{4} \hat{\mathbf{D}} \cdot \frac{\partial}{\partial \mathbf{r}_{c}} \ln \Psi, \\
& \overline{\mathbf{\Omega}}=\left(\boldsymbol{\Omega}_{11}-\boldsymbol{\Omega}_{22}\right)+\left(\boldsymbol{\Omega}_{21}-\boldsymbol{\Omega}_{21}^{T}\right), \\
& \overline{\mathbf{D}}=k_{B} T \overline{\boldsymbol{\Omega}}, \\
& \hat{\mathbf{D}}=\left(\mathbf{D}_{11}+\mathbf{D}_{22}\right)+\left(\mathbf{D}_{21}+\mathbf{D}_{21}^{T}\right), \\
& \mathbf{v}_{c}=\frac{1}{2}\left(\mathbf{v}_{1}+\mathbf{v}_{2}\right),
\end{aligned}
$$

where we have made use of Eqs. (3) and (18). Multiplying Eq. (43) by $\Psi$ and integrating over the internal coordinates, one can show that

$$
\begin{aligned}
\mathbf{j}_{c}= & \frac{1}{2}\left[2\left\langle\mathbf{v}_{c}\right\rangle+\left\langle\overline{\mathbf{\Omega}} \cdot \mathbf{f}^{s}\right\rangle\right] C-\frac{1}{4}\left[2\left\langle\frac{\partial}{\partial \mathbf{q}} \cdot\left(\mathbf{D}_{11}-\mathbf{D}_{22}\right)\right\rangle\right. \\
& \left.-\left\langle\frac{\partial}{\partial \mathbf{r}_{c}} \cdot\left(\mathbf{D}_{11}+\mathbf{D}_{22}\right)\right\rangle+\frac{\partial}{\partial \mathbf{r}_{c}} \cdot\langle\hat{\mathbf{D}}\rangle\right] C-\frac{1}{4}\langle\hat{\mathbf{D}}\rangle \cdot \frac{\partial C}{\partial \mathbf{r}_{c}} .
\end{aligned}
$$

The steady-state solution $\left(\mathbf{j}_{c}=0\right)$ of Eq. (39) using Eq. (48) and appropriate boundary conditions gives the steady-state mass distribution $C\left(\mathbf{r}_{c}\right)$ in physical space. Note that the center-of-mass distributions presented in Sec. III represent numerical solutions to this same problem for a much more complicated model of the molecule.

Equation (48) is a general dilute solution result for a Brownian bead-spring dumbbell in an arbitrary flow field and geometry. We refer to the terms proportional to $C$ as "drift" contributions and the term proportional to the gradient of $C$ as a "diffusive" contribution. The first line in Eq. (48) represents a deterministic contribution to the drift term, while the second line is a Brownian contribution to the drift term. The last line represents standard Fickian diffusionnote that $\langle\hat{\mathbf{D}}\rangle / 4$ is the Kirkwood diffusivity, ${ }^{7}$ averaged over the internal coordinates of the molecule. Equation (48) indicates that a Brownian molecule in rectilinear flow in a confined geometry can experience a drift normal to the imposed streamlines, and this drift can have both deterministic and Brownian contributions. Both of these mechanisms have been discussed in the literature, which we will review shortly. First we give a brief physical explanation of the drift terms appearing in Eq. (48).

For rectilinear flow, the $\left\langle\mathbf{v}_{c}\right\rangle C$ term represents pure convection along imposed streamlines. The $\left\langle\boldsymbol{\Omega} \cdot \mathbf{f}^{s}\right\rangle C$ term indicates that a dumbbell in tension (or compression) near a solid boundary can experience a deterministic drift which is not necessarily along the imposed streamlines. The three drift terms appearing in the second line of Eq. (48) are Brownian in nature and arise from spatial variations in the hydrodynamic interactions. The first two of these terms are due to spatial variations in bead mobility, and vanish when wall contributions to the hydrodynamic interaction tensor are ignored. The third is due to spatial variations in bead mobility as well as spatial variations in bead-bead hydrodynamic interactions. For the special case of homogeneous rectilinear flow, Eq. (48) becomes

$$
\begin{aligned}
\mathbf{j}_{c}^{\text {homo }}= & \frac{1}{2}\left[2\left\langle\mathbf{v}_{c}\right\rangle+\left\langle\overline{\mathbf{\Omega}} \cdot \mathbf{f}^{\mathrm{s}}\right\rangle\right] C-\frac{1}{2}\left\langle\frac{\partial}{\partial \mathbf{q}} \cdot \overline{\mathbf{D}}\right\rangle C \\
& -\frac{1}{4}\langle\hat{\mathbf{D}}\rangle \cdot \frac{\partial C}{\partial \mathbf{r}_{c}} .
\end{aligned}
$$

In the absence of wall hydrodynamic interactions, Eq. (49) predicts no migration for homogeneous rectilinear flow.

Equation (39) has been the starting point for many investigations into migration effects in flowing dilute solutions of long flexible polymers. Although some investigations ${ }^{11-14,54}$ have employed bulk hydrodynamic interactions (Oseen-Burgers), many have ignored hydrodynamic interactions altogether. We are aware of only one qualitative discussion ${ }^{14}$ considering a simple first-order account of wall hydrodynamic interaction for a flexible polymer chain.

\section{B. Chain migration neglecting wall hydrodynamic interaction}

The use of Oseen-Burgers (OB) free-solution hydrodynamics allows considerable simplification of Eq. (48). In free-solution $\boldsymbol{\Omega}_{i i}=$ constant and $\boldsymbol{\Omega}_{i j}$ is symmetric, yielding ${ }^{11}$ 


$$
\begin{aligned}
\mathbf{j}_{c}^{\mathrm{OB}}= & {\left[\left\langle\mathbf{v}_{c}\right\rangle-\frac{k_{B} T}{2} \frac{\partial}{\partial \mathbf{r}_{c}} \cdot\left\langle\boldsymbol{\Omega}_{12}^{\mathrm{OB}}\right\rangle\right] C } \\
& -\frac{k_{B} T}{2 \zeta}\left\langle\mathbf{I}+\zeta \boldsymbol{\Omega}_{12}^{\mathrm{OB}}\right\rangle \cdot \frac{\partial C}{\partial \mathbf{r}_{c}} .
\end{aligned}
$$

Deterministic cross-stream migration in rectilinear flow is not predicted by this model, although there is cross-stream Brownian drift due to coupling of hydrodynamics with spatial variations in the distribution of polymer configurations.

Sekhon et al. considered the case of bulk hydrodynamic interactions [Eq. (50)] in rectilinear slit flow, $v_{x}\left(y_{c}\right)$ $=f\left(y_{c}\right)$, where $y_{c}$ is the distance from the centerline. In this case $\left\langle\boldsymbol{\Omega}_{12}^{\mathrm{OB}}\right\rangle$ can be written in the general form

$$
\left\langle\boldsymbol{\Omega}_{12}^{\mathrm{OB}}\right\rangle\left(y_{c}\right)=\left(\begin{array}{ccc}
a_{11}\left(y_{c}\right) & a_{12}\left(y_{c}\right) & 0 \\
a_{12}\left(y_{c}\right) & a_{22}\left(y_{c}\right) & 0 \\
0 & 0 & a_{33}\left(y_{c}\right)
\end{array}\right) .
$$

At steady state $C$ is a function of $y_{c}$ only, and the steadystate wall-normal component of $\mathbf{j}_{c}^{\mathrm{BHI}}$ is given by

$$
\begin{aligned}
j_{c y}^{\mathrm{OB}}= & -\frac{k_{B} T}{2} \frac{\partial a_{22}\left(y_{c}\right)}{\partial y_{c}} C\left(y_{c}\right) \\
& -\frac{k_{B} T}{2 \zeta}\left(1+\zeta a_{22}\left(y_{c}\right)\right) \frac{\partial C\left(y_{c}\right)}{\partial y_{c}} \\
= & 0,
\end{aligned}
$$

from which Sekhon et al. concluded that cross-stream migration is possible. Although Sekhon et al. stopped here, we can continue the analysis a bit further. We rewrite Eq. (52) as

$$
\frac{\partial \ln C}{\partial y_{c}}=-\zeta\left(1+\zeta a_{22}\right)^{-1} \frac{\partial a_{22}}{\partial y_{c}},
$$

and note that for Oseen-Burgers hydrodynamic interactions $a_{22} \propto q^{-1}\left(1+q_{y}^{2} / q^{2}\right)$, where $q=|\mathbf{q}|$. Considering the flow to be parabolic, one can readily deduce that $a_{22}$ is always positive and decreases monotonically from a maximum at the center line. The use of bulk hydrodynamic interactions therefore (incorrectly) predicts migration toward the walls, leading to a minimum in $C\left(y_{c}\right)$ at the center line of the channel (note that $\partial a_{22} / \partial y_{c}=0$ at the center line of the channel). $C\left(y_{c}\right)$ increases monotonically toward the channel walls. This prediction compares well to the center of mass distribution in Fig. 11(b), obtained from our detailed chain simulations ignoring the wall correction to the hydrodynamic interactions. Brunn ${ }^{12,13}$ numerically solved for the steady-state $C\left(y_{c}\right)$ for a dumbbell through a first-order perturbation analysis using Oseen-Burgers free-solution hydrodynamic interactions and also found migration toward the walls. Interestingly, Brunn also found that using preaveraged OseenBurgers hydrodynamics predicted depletion at the walls.

Ignoring hydrodynamic interactions altogether $\left(\boldsymbol{\Omega}_{i i}\right.$ $=\boldsymbol{\Omega}_{i j}=0$ ), but assuming a configuration dependent friction coefficient (CDFC), ${ }^{55} \zeta=\zeta_{o} g(q)$ gives $^{56}$

$$
\begin{aligned}
\mathbf{j}_{c}^{\mathrm{CDFC}}= & {\left[\left\langle\mathbf{v}_{c}\right\rangle-\frac{k_{B} T}{2 \zeta_{o}} \frac{\partial}{\partial \mathbf{r}_{c}} \cdot\left\langle g^{-1}(q)\right\rangle\right] C } \\
& -\frac{k_{B} T}{2 \zeta_{o}}\left\langle g^{-1}(q)\right\rangle \cdot \frac{\partial C}{\partial \mathbf{r}_{c}},
\end{aligned}
$$

and the steady-state concentration is determined from

$$
\frac{\partial \ln C}{\partial y_{c}}=-\frac{1}{\left\langle g^{-1}(q)\right\rangle} \frac{\partial\left\langle g^{-1}(q)\right\rangle}{\partial y_{c}} .
$$

Since the friction factor $g$ is positive and generally ${ }^{55}$ taken to be proportional to $q,\left\langle g^{-1}\right\rangle$ is again a maximum at the center line and decreases toward the wall, giving the same qualitative results as obtained from the use of bulk hydrodynamic interactions. This is not a coincidence; if we ignore wall hydrodynamic interaction in Eq. (48), the steady-state mass flux in the wall-normal direction results in

$$
\frac{\partial \ln C}{\partial y_{c}}=-\frac{1}{\langle\hat{\mathbf{D}}\rangle_{y y}} \frac{\partial\langle\hat{\mathbf{D}}\rangle_{y y}}{\partial y_{c}},
$$

where $\hat{\mathbf{D}}(\mathbf{q})$ is proportional to the Kirkwood diffusivity of the dumbbell. The diffusivity of the dumbbell decreases with increasing $q$ whether we use bulk hydrodynamics or the CDFC model. We note here that Seo et al. ${ }^{54}$ used a preaveraging for the configuration-dependent friction coefficient model and predicted the opposite-depletion at the wall. Note also that if one uses a friction coefficient that decreases with increasing $q$, Eq. (56) predicts depletion near the wall.

Finally, ignoring hydrodynamic interactions altogether and assuming a constant bead friction coefficient, gives the FD model result

$$
\mathbf{j}_{c}^{\mathrm{FD}}=\left\langle\mathbf{v}_{c}\right\rangle C-\frac{k_{B} T}{2 \zeta} \frac{\partial C}{\partial \mathbf{r}_{c}},
$$

which predicts no cross-stream migration in rectilinear flow. This prediction is consistent with the results of Fig. 11(d), the mass distribution for a free-draining chain.

\section{Cross-stream migration with wall hydrodynamic interaction}

We now turn our attention back to the general expression for the center of mass flux [Eq. (48)], and incorporate the hydrodynamic effect of walls on chain migration. For rectilinear channel flow, the deterministic (DET) portion of $\mathbf{j}_{c}$ in the wall-normal direction is given by

$$
j_{c y}^{\mathrm{DET}}=\left\langle\overline{\mathbf{\Omega}} \cdot \mathbf{f}^{\mathrm{s}}\right\rangle_{y}\left(y_{c}\right) C\left(y_{c}\right) .
$$

Recall that $\overline{\mathbf{\Omega}}$ [Eq. (44)] is proportional to the difference in bead mobilities and also proportional to the asymmetric portion of the hydrodynamic interaction between the two beads, $\boldsymbol{\Omega}_{12}-\boldsymbol{\Omega}_{12}^{T}$. Recall also that $\overline{\boldsymbol{\Omega}}=0$ when wall hydrodynamic interaction is ignored. Now suppose for the moment we ignore the Brownian drift terms in Eq. (48) and allow the deterministic and Fickian terms to balance at steady state yielding 


$$
\frac{\partial \ln C}{\partial y_{c}}=\left\langle\overline{\mathbf{\Omega}} \cdot \mathbf{f}^{s}\right\rangle_{y} .
$$

To get an idea of the magnitude and sign of the deterministic contribution to migration, we consider rectilinear shear flow over a single infinite plane wall. As mentioned in Appendix A, for the case of point force hydrodynamics near an infinite plane wall, an analytical expression is available for the hydrodynamic interaction tensor, $\boldsymbol{\Omega}=\boldsymbol{\Omega}_{\mathrm{PW}}^{\mathrm{PF}}$. We can use the simple dumbbell model along with $\boldsymbol{\Omega}_{\mathrm{PW}}^{\mathrm{PF}}$ to gain some insight into the origins of the wall-normal drift. The Green's function for a point force located at $\mathbf{x}_{j}=\left(x_{j}, y_{j}, z_{j}\right)^{T}$ a distance $y_{j}$ above an infinite plane wall can be expressed as ${ }^{29}$

$$
\begin{aligned}
\boldsymbol{\Omega}_{\mathrm{PW}}^{\mathrm{PF}}\left(\mathbf{x}, \mathbf{x}_{j}\right)= & \boldsymbol{\Omega}^{\mathrm{PF}}\left(\mathbf{x}-\mathbf{x}_{j}\right)-\boldsymbol{\Omega}^{\mathrm{PF}}\left(\mathbf{x}-\hat{\mathbf{x}}_{j}\right)-2 y_{j} \boldsymbol{\Omega}^{\mathrm{SD}}\left(\mathbf{x}-\hat{\mathbf{x}}_{j}\right) \\
& +2 y_{j}^{2} \boldsymbol{\Omega}^{\mathrm{PD}}\left(\mathbf{x}-\hat{\mathbf{x}}_{j}\right),
\end{aligned}
$$

where $\hat{\mathbf{x}}_{j}=\left(x_{j},-y_{j}, z_{j}\right)^{T}$ is the position of the "image" of particle $j$. The first two terms on the right are the point force solutions for the particle and its image in free solution, while the last two terms involve the image Stokeslet doublet $\boldsymbol{\Omega}^{\mathrm{SD}}$ and image potential dipole $\boldsymbol{\Omega}^{\mathrm{PD}}$ in free solution. The hydrodynamic interaction tensor is then given by

$$
\boldsymbol{\Omega}_{i j}=\left\{\begin{array}{cc}
\boldsymbol{\Omega}_{\mathrm{PW}}^{\mathrm{PF}}\left(\mathbf{r}_{i}, \mathbf{r}_{j}\right) & \text { for } i \neq j, \\
\boldsymbol{\Omega}_{\mathrm{PW}}^{\mathrm{PF}}\left(\mathbf{r}_{i}, \mathbf{r}_{j}\right)-\mathbf{\Omega}^{\mathrm{PF}}\left(\mathbf{r}_{i}, \mathbf{r}_{j}\right) & \text { for } i=j .
\end{array}\right.
$$

Jhon and Freed ${ }^{14}$ have also considered the deterministic contribution to the drift of a dumbbell in rectilinear flow near a wall. Those authors used the first two terms of Eq. (60) (the point force and its image) to obtain a qualitative picture of the hydrodynamic drift of the dumbbell center of mass. Ignoring the last two terms in Eq. (60) gives a "Green's function" that violates the reciprocity requirement for the Green's function of a self-adjoint operator $\left(\boldsymbol{\Omega}_{i j} \neq \boldsymbol{\Omega}_{j i}^{T}\right)$. This is in essence an artificial symmetry breaking, whose consequences are unknown. The work presented here for a dumbbell near an infinite plane wall (as well as the general method outlined in earlier sections) does satisfy the identity $\boldsymbol{\Omega}_{i j}$ $=\boldsymbol{\Omega}_{j i}^{T}$. Jhon and Freed ${ }^{14}$ did qualitatively state that drift away from the wall was possible, but did not give specific examples of how this drift depended on intramolecular configuration and position relative to the wall. Here, we address these issues directly.

In simple shear, the flexible polymer stretches and "flips" repeatedly. For the case of an infinite plane wall, the wall-normal drift of our dumbbell model can be characterized by three variables - the magnitude of the connector vector $(q)$, the angle $(\theta)$ that the connector vector makes with the wall, and the distance $\left(y_{1}\right)$ of bead 1 from the wall. This representation is illustrated in Fig. 12.

Using this crude model, we can easily compute how the magnitude and sign of $\left(\overline{\mathbf{\Omega}} \cdot \mathbf{f}^{\mathrm{s}}\right)_{y}$ depends on molecular extension, orientation relative to the wall, and distance from the wall. The results are illustrated in Fig. 13, which shows $v_{c, y}=\left(\overline{\mathbf{\Omega}} \cdot \mathbf{f}^{\mathrm{s}}\right)_{y}$ as a function of $y_{1}$ for various molecular orientations and extensions. We only show results for values of $0 \leqslant \theta \leqslant \pi / 2$ due to symmetry; $v_{c, y}\left(q, \theta, y_{1}\right)=v_{c, y}(q, \pi$ $\left.-\theta, y_{1}\right)$ for the dumbbell model. In these results the dumbbell corresponds to one spring from our DNA model (spring

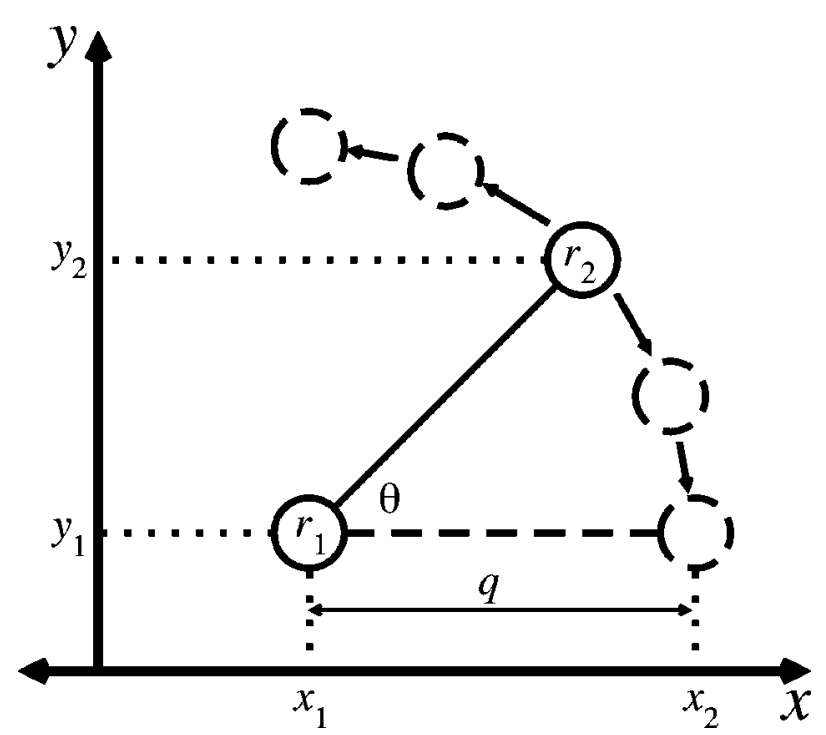

FIG. 12. Model system used to obtain the results in Fig. 13.

contour length $q_{o} \approx 2.1 \mu \mathrm{m}$ ) with no excluded volume. Figure 13(a) shows $v_{c, y}$ as a function of $y_{1}$ for a dumbbell oriented parallel to the wall for different values of $q$. For this particular orientation, the drift is always away from the wall. Figures 13(b)-13(d) show results for increasing values of $\theta$. As $\theta$ increases, drift towards the wall is observed for dumbbells close enough to the wall. When $\theta$ becomes large enough, the drift is always toward the wall. Recall that $\overline{\mathbf{\Omega}}$ [Eq. (44)] is proportional to the mobility difference between the two beads of the dumbbell (this contributes a drift toward the wall), and also proportional to the non-symmetric portion of the hydrodynamic interaction between the two beads (this contributes a drift away from the wall); the direction of the deterministic migration is determined from a competition between these two terms. The orientations that give rise to a negative $v_{c, y}$ are not particularly relevant for the case of simple shear near a plane wall, as $\langle\theta\rangle$ is fairly small for $\dot{\gamma}_{\text {eff }} \lambda_{1}>1\left(\langle\theta\rangle \rightarrow 0\right.$ as $\left.\dot{\gamma}_{\text {eff }} \rightarrow \infty\right)$. Even during the flipping pro-

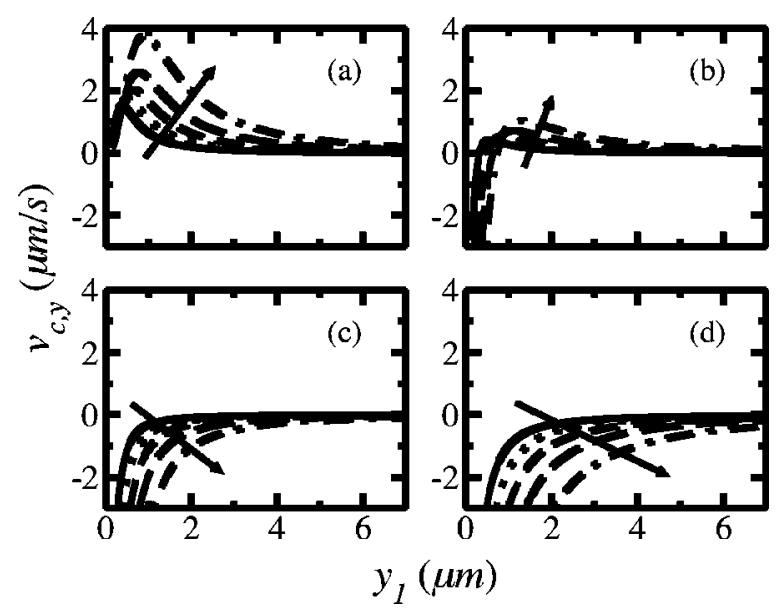

FIG. 13. Wall-normal center of mass drift as a function of distance from the wall for various values of molecular extension and orientation: (a) $\theta=0$, (b) $\theta=\pi / 8$, (c) $\theta=\pi / 4$, and (d) $\theta=\pi / 2$. The arrow indicates direction of increasing $q$; values of $q=\{0.3,0.4,0.5,0.6,0.7\} q_{o}$ are shown. For this model, $q_{o}=2.1 \mu \mathrm{m}$ and $\left\langle q^{2}\right\rangle_{\mathrm{eq}}^{1 / 2} \approx 0.225 q_{o}$. 


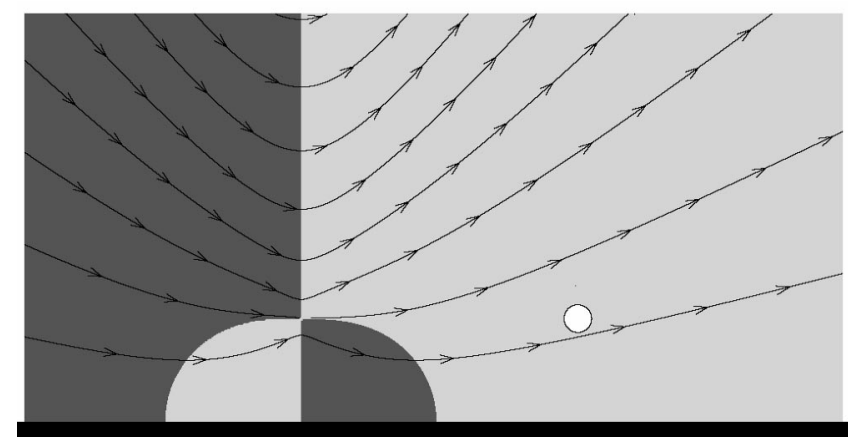

FIG. 14. The velocity field due to a point force located at $(x, y)=$ $(-5 a, 4 a)$ acting in the positive $x$ direction. The lines correspond to streamlines, while the light and dark areas indicate regions where the wall-normal velocity is positive (away from wall) and negative (towards the wall), respectively. Also shown is a "bead" of radius a located at $(x, y)=(5 a, 4 a)$-this can be thought of as the other end of a relaxing dumbbell oriented parallel to the wall.

cess when $\theta$ passes through $\pi / 2$ (orientation perpendicular to the wall), the magnitude of $q$, and therefore $\mathbf{f}^{s}$, is small; the magnitude of $v_{c, y} \rightarrow 0$ as $\left|f^{s}\right| \rightarrow 0$. Figure 14 shows the wallnormal component of the velocity field due to a point force acting parallel to an infinite plane wall. This can be interpreted as the wall-normal flow set up by one end of a relaxing dumbbell oriented parallel to the wall. The other end of the dumbbell (illustrated as a white circle of radius $a$ ) experiences a drift away from the wall because it lies in a region where the flow has a component away from the wall.

From the preceding paragraph it is clear that for rectilinear channel flow $\left(\overline{\boldsymbol{\Omega}} \cdot \mathbf{f}^{s}\right)_{y}$ is positive near the wall and decreases toward the center line of the channel, and from Eq. (59) we conclude that deterministic migration will be toward the center line when full wall hydrodynamic interactions are considered. The results of Sec. III B demonstrate that for the channel sizes and chain lengths considered in this study, the deterministic migration is the dominant contribution and is directed away from the wall. In simple physical terms, the wall-normal flow induced by a horizontally oriented point force near a wall is the origin of the migration seen in our simulations.

For completeness, we conclude with an analysis of the RM model [Eq. (20)]. For this model Eq. (48) can be written as

$$
\begin{aligned}
\mathbf{j}_{c}^{\mathrm{RM}}= & \frac{1}{2}\left[2\left\langle\mathbf{v}_{c}\right\rangle+\left\langle\left(\boldsymbol{\Omega}_{11}-\mathbf{\Omega}_{22}\right) \cdot \mathbf{f}^{s}\right\rangle\right] C+\frac{1}{4}\left[2\left\langle\frac{\partial}{\partial \mathbf{r}_{1}} \cdot \mathbf{D}_{11}\right\rangle\right. \\
& \left.+2\left\langle\frac{\partial}{\partial \mathbf{r}_{2}} \cdot \mathbf{D}_{22}\right\rangle-\frac{\partial}{\partial \mathbf{r}_{1}} \cdot\left\langle\mathbf{D}_{11}\right\rangle-\frac{\partial}{\partial \mathbf{r}_{2}} \cdot\left\langle\mathbf{D}_{22}\right\rangle\right] C \\
& -\frac{1}{4}\left\langle\mathbf{D}_{11}+\mathbf{D}_{22}\right\rangle \cdot \frac{\partial C}{\partial \mathbf{r}_{c}}
\end{aligned}
$$

We already know that deterministic migration due to differences in bead mobility is always toward the wall. However, near the wall the local shear rate is high and the difference in bead mobilities is therefore small; deterministic migration to the wall is weak. Since bead mobilities decrease toward the wall, the first two terms in the Brownian contribution to mi- gration in Eq. (62) yield migration toward the center line, while the last two terms yield migration toward the wall. If one assumes that $\left|\left\langle\partial / \partial \mathbf{r}_{i} \cdot \mathbf{D}_{i i}\right\rangle\right| \approx\left|\partial / \partial \mathbf{r}_{i} \cdot\left\langle\mathbf{D}_{i i}\right\rangle\right|$ then if migration occurs at all, it should be toward the center line. This simple analysis agrees qualitatively with the mass distribution in Fig. 11(c), obtained from our detailed simulations of a reduced mobility chain. The weak migration effect in Fig. 11(c) indicates that spatial dependence of the bead mobilities plays only a minor role in migration of flexible polymers in rectilinear channel flow.

\section{CONCLUSIONS}

We have performed simulations of DNA (contour length $\approx 10-126 \mu \mathrm{m}$ ) using a model that includes excluded volume and hydrodynamic interactions in a confined geometry. The behavior of these DNA chains was examined in pressure-driven flow through micron-scale channels (channel width $\approx 1-10 \mu \mathrm{m})$.

The steady-state stretch of DNA chains was examined as a function of channel width and flow strength. The flow strength needed to stretch a chain away from its equilibrium length was shown to increase with decreasing channel width. This is fairly well explained using a simple blob picture.

The mass distribution of the DNA chains in the cross section of the channel was examined as a function of molecular weight, channel width, and flow strength. A significant hydrodynamically induced depletion of DNA was observed near the wall. The thickness of the depletion layer was shown to increase with increasing molecular weight. The result is that higher molecular weight chains are more concentrated in the center of the channel where the imposed axial fluid velocity is higher. This causes higher molecular weight chains to move with a higher average axial velocity than lower molecular weight chains, resulting in separation based on molecular weight. This hydrodynamic depletion effect may be responsible for an unexpected phenomenon observed in the simulations: at least for some shear rates, the degree of stretch of chains does not increase monotonically from the center line to the channel walls - the more highly stretched chains may migrate, leaving behind the less stretched ones. Future work will test this idea.

From kinetic theory analysis of a dumbbell model of a confined polymer and simulations of the more complex multibead model, we draw the following qualitative conclusions for dilute flexible polymer solutions in inhomogeneous rectilinear channel flow:

(1) There is a "deterministic" hydrodynamic drift away from the walls due to the wall contribution to the hydrodynamic interactions. A horizontally oriented point force near a wall generates a wall-normal velocity "ahead" of it. This effect is strongest near walls due both to proximity to the wall and high local shear rate (and therefore polymer extension and spring force).

(2) There is a Brownian drift (due to coupling of the hydrodynamic interactions with spatial variations in the distribution of polymer configurations) away from the center line.

(3) The balance of these two effects, along with Fickian diffusion, determines the steady-state concentration profile. 
This balance in general leads to a nonmonotonic concentration profile across the channel.

Our full model results indicate that, for our chain lengths and channel widths, the competition between deterministic drift (wall hydrodynamics) and Brownian drift result in a minimum in concentration at the center line, a maximum in concentration near the centerline, followed by a monotonically decreasing concentration toward the walls with substantial depletion of polymer concentration occurring toward the walls. The depletion layer grows with increasing flow strength and increasing molecular weight.

\section{ACKNOWLEDGMENTS}

The authors gratefully acknowledge the support of the NSF Nanoscale Modeling and Simulation Program, and the Department of Energy.

\section{APPENDIX A: APPROXIMATE RPY HYDRODYNAMICS NEAR AN INFINITE PLANE WALL}

As discussed in the text, the use of point force hydrodynamics can lead to nonphysical behavior, in particular nonpositive semidefiniteness of the diffusion tensor. This occurs when "beads" (which represent many monomer units) of the chain overlap. The overlap itself is not unphysical, and in free solution the point force, or Oseen-Burgers, formulation is typically replaced by RPY hydrodynamics. The free solution RPY formulation contains a near field "fix" which guarantees positive semidefiniteness of the diffusion tensor for all chain configurations. The method presented in this work meets this practical requirement as well as satisfying the reciprocity requirement $\boldsymbol{\Omega}\left(\mathbf{x}_{1}, \mathbf{x}_{2}\right)=\boldsymbol{\Omega}^{T}\left(\mathbf{x}_{2}, \mathbf{x}_{1}\right)$, and can be considered as an approximate RPY formulation for confined geometries. We will now give an example of our formulation for the special case of infinite plane wall.

For nonoverlapping beads, the free-solution RPY tensor can be written as

$$
\boldsymbol{\Omega}^{\mathrm{RPY}}=\boldsymbol{\Omega}^{\mathrm{PF}}-\frac{2 a^{2}}{3} \boldsymbol{\Omega}^{\mathrm{PD}}
$$

where $\boldsymbol{\Omega}^{\mathrm{PF}}$ and $\boldsymbol{\Omega}^{\mathrm{PD}}$ are the hydrodynamic interaction tensors for a point force (Oseen-Burgers) and potential dipole, respectively. ${ }^{41}$ In the presence of an infinite plane wall, we can write

$$
\boldsymbol{\Omega}_{\mathrm{PW}}^{\mathrm{RPY}}=\boldsymbol{\Omega}_{\mathrm{PW}}^{\mathrm{PF}}-\frac{2 a^{2}}{3} \boldsymbol{\Omega}_{\mathrm{PW}}^{\mathrm{PD}},
$$

where the subscript PW indicates that these hydrodynamic interaction tensors are now evaluated in the presence of a single infinite plane wall; $\boldsymbol{\Omega}_{\mathrm{PW}}^{\mathrm{RPY}}$ can be considered an approximate RPY hydrodynamic interaction tensor for a particle near an infinite plane. Note that our method is a generalization of this to arbitrary geometries. There are analytical solutions ${ }^{41}$ for both $\boldsymbol{\Omega}_{\mathrm{PW}}^{\mathrm{PF}}$ and $\boldsymbol{\Omega}_{\mathrm{PW}}^{\mathrm{PD}}$. The $\boldsymbol{\Omega}_{\mathrm{PW}}^{\mathrm{PF}}$ term satisfies the reciprocity requirement, but the potential dipole term does not (it is not a point force solution). However, the effect is weak. Consider a point force located at $r_{1}=(0,2 a, 0)$ above an infinite plane (plane at $y=0$ ), and another point $r_{2}=(x, 4 a, 0)$, as illustrated in Fig. 15. Figure 16(a) compares

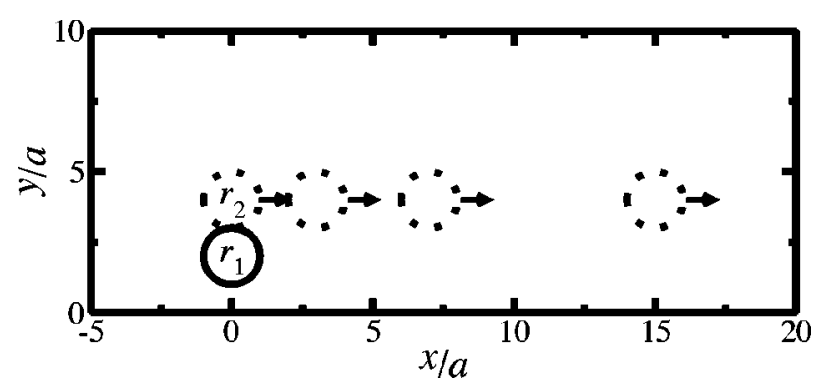

FIG. 15. Example system from which the results in Fig. 16 were obtained.

the $y y$ component of $\boldsymbol{\Omega}_{\mathrm{PW}}^{\mathrm{RPY}}\left(r_{1}, r_{2}\right), \quad\left(\boldsymbol{\Omega}_{\mathrm{PW}}^{\mathrm{RPY}}\left(r_{1}, r_{2}\right)\right.$ $\left.+\left[\boldsymbol{\Omega}_{\mathrm{PW}}^{\mathrm{RPY}}\left(r_{2}, r_{1}\right)\right]^{T}\right) / 2$ [enforcement of reciprocity using Eq. (19)] and $\boldsymbol{\Omega}_{\mathrm{PW}}^{\mathrm{PF}}$ as a function of $x$. Likewise, Figs. 16(b) and 16(c) show the $y x$ and $x x$ components. Two things are clear from Fig. 16. First, the potential dipole term is a near field modification of the point force solution. Second, the "nonreciprocal" portion ( $\left.\left[\boldsymbol{\Omega}\left(\mathbf{x}_{1}, \mathbf{x}_{2}\right)-\boldsymbol{\Omega}^{T}\left(\mathbf{x}_{2}, \mathbf{x}_{1}\right)\right] / 2\right)$ of the potential dipole term is short ranged and very weak (it is difficult to tell the difference between the solid and dotted lines).

Our development of the general method outlined in the text was guided by the observations described above for a particle near an infinite plane. It worth pointing out that we could satisfy the reciprocity requirement by getting the higher order terms from a multipole expansion for two spheres near an infinite plane satisfying no-slip on the spheres and the plane. However, since we are interested in a method which is applicable to general geometries, we choose the approximate RPY formulation described in this paper. Note also that a multipole expansion would not address the issue of "bead"-" "bead" overlap, which is accounted for in our approximate RPY formulation.

\section{APPENDIX B: POSITIVE-SEMIDEFINITENESS OF THE DIFFUSION TENSOR}

The formulation presented in Appendix A for a particle near an infinite plane wall ensures a positive-semidefinite diffusion tensor for all chain configurations provided the bead "surfaces" do not overlap with each other or with the

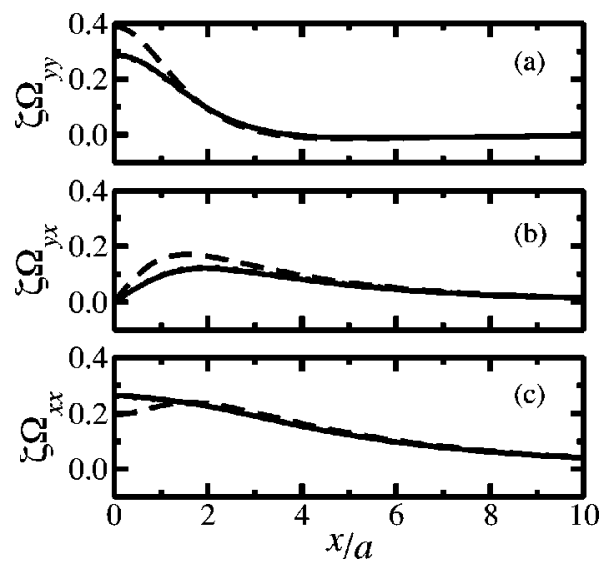

FIG. 16. Comparison between $\boldsymbol{\Omega}_{\mathrm{PW}}^{\mathrm{RPY}}\left(r_{1}, r_{2}\right)$ (solid line), $\left[\boldsymbol{\Omega}_{\mathrm{PW}}^{\mathrm{RPY}}\left(r_{1}, r_{2}\right)\right.$ $\left.+\left[\boldsymbol{\Omega}_{\mathrm{PW}}^{\mathrm{RPY}}\left(r_{2}, r_{1}\right)\right]^{T}\right] / 2$ (dotted line), and $\boldsymbol{\Omega}_{\mathrm{PW}}^{\mathrm{PF}}\left(r_{1}, r_{2}\right)$ (dashed line): (a) $y y$ component, (b) $y x$ component, and (c) $x x$ component. 
wall. When the free solution portion ${ }^{41}$ of $\boldsymbol{\Omega}_{\mathrm{PW}}^{\mathrm{RPY}}$ is replaced with the equivalent RPY near field modification [Eq. (6) with Eq. (9)], the resulting diffusion tensor is guaranteed to remain positive semidefinite for all chain configurations, provided the bead surfaces do not penetrate the wall. In contrast, the general method outlined in the text generates a positivesemidefinite diffusion tensor for all chain configurations (provided, of course, that the interaction sites of the chain remain within the proscribed geometry). However, positive semidefiniteness is only guaranteed to the level of accuracy of the Stokes flow problem which generated the hydrodynamic interaction tensor. As mentioned in Sec. II, in this work all entries in the diffusion tensor which were less then $0.1 \%$ of the maximum diagonal component of the diffusion tensor were set to zero, which seemed to keep the accuracy of the diffusion tensor consistent with the accuracy of the Stokes flow problem (for our choice of parameters). For robustness, one may wish to guarantee positive semidefiniteness of the diffusion tensor even in the event that accuracy of the Stokes flow problem is insufficient. In the following we address this issue.

As mentioned above, the Oseen-Burgers formulation is not guaranteed to remain positive semidefinite for all chain configurations. The reason for this is that the Green's function for a point force (in any geometry) is a "far-field" solution. For example, the velocity field at $\mathbf{x}^{\nu}$ due to a point force acting at $\mathbf{x}^{\mu}$ is given by

$$
\mathbf{v}_{\mu}^{\nu}=\frac{1}{k_{B} T} \mathbf{D}^{\nu \mu} \cdot \mathbf{f}^{\mu},
$$

which diverges as $\left|\mathbf{x}^{\nu}-\mathbf{x}^{\mu}\right|^{-1}$ in free solution; in the OseenBurgers formulation, the velocity is infinite at the location of the point force. However, the velocity of the bead located at $\mathbf{x}^{\nu}$ is defined through the Stokes drag

$$
\mathbf{v}_{\mu}^{\mu}=\frac{1}{k_{B} T} \mathbf{D}^{\mu \mu} \cdot \mathbf{f}^{\mu} .
$$

In free solution, the velocity of bead $\mu$ is a constant for a given force (independent of position). Note that for two beads close enough together, the use of Oseen-Burgers hydrodynamics results in $\left|\mathbf{v}_{\mu}^{\nu}\right|>\left|\mathbf{v}_{\mu}^{\mu}\right|$; the motion of bead $\mu$ generates a solvent velocity greater than the velocity of bead $\mu$. This unphysical "negative viscous dissipation" leads to a non-positive-semidefinite diffusion tensor. As we now demonstrate, it is fairly simple to check if hydrodynamic interaction between two particular beads results in negative viscous dissipation.

The velocity of bead $\mu$ is given by Eq. (B2), while the velocity at the location of bead $\nu$ due to motion of bead $\mu$ is given by Eq. (B1). The magnitude squared of these velocities is given by

$$
\begin{aligned}
& \left(k_{B} T\right)^{2}\left|\mathbf{v}_{\mu}^{\mu}\right|^{2}=\mathbf{f}^{\mu} \cdot\left(\mathbf{D}^{\mu \mu}\right)^{2} \cdot \mathbf{f}^{\mu}, \\
& \left(k_{B} T\right)^{2}\left|\mathbf{v}_{\mu}^{\nu}\right|^{2}=\mathbf{f}^{\mu} \cdot\left(\mathbf{D}^{\nu \mu}\right)^{2} \cdot \mathbf{f}^{\mu},
\end{aligned}
$$

where $\mathbf{v}_{\mu}^{\mu}$ is the velocity of bead $\mu$ due to a point force on bead $\mu$, and $\mathbf{v}_{\mu}^{\nu}$ is the solvent velocity at bead $\nu$ due to the point force at bead $\mu$. Taking the difference and requiring positive viscous dissipation, we obtain

$$
\left(k_{B} T\right)^{2}\left(\left|\mathbf{v}_{\mu}^{\mu}\right|^{2}-\left|\mathbf{v}_{\mu}^{\nu}\right|^{2}\right)=\mathbf{f}^{\mu} \cdot\left[\left(\mathbf{D}^{\mu \mu}\right)^{2}-\left(\mathbf{D}^{\nu \mu}\right)^{2}\right] \cdot \mathbf{f}^{\mu} \geqslant 0 .
$$

This is the definition of positive semidefiniteness for the 3 $\times 3$ matrix $\left(\mathbf{D}^{\mu \mu}\right)^{2}-\left(\mathbf{D}^{\nu \mu}\right)^{2}$. We can easily check for positive semidefiniteness (eigenvalues of a $3 \times 3$ matrix), and determine if $\mathbf{D}^{\nu \mu}$ (the hydrodynamic interaction tensor for a point force at $\mu$ ) needs to be modified. If $\left(\mathbf{D}^{\mu \mu}\right)^{2}-\left(\mathbf{D}^{\nu \mu}\right)^{2}$ is not positive semidefinite, the simplest modification is to simply set $\mathbf{D}^{\nu \mu}=\mathbf{D}^{\mu \mu}$. Physically, this means that in the absence of any other force acting on bead $\nu$, bead $\nu$ moves with the velocity of bead $\mu$. Note that this gives a symmetric hydrodynamic interaction tensor. For computational efficiency, if $\left(\mathbf{D}^{\mu \mu}\right)^{2}-\left(\mathbf{D}^{\nu \mu}\right)^{2}$ is not positive semidefinite, one may set $\mathbf{D}^{\nu \mu}=c \mathbf{D}^{\mu \mu}$, where $c<1$.

The procedure outlined above is fairly straightforward, but there is a sticking point. We can repeat the above argument for a point force acting on bead $\nu$, rather than bead $\mu$. The result is

$$
\left(k_{B} T\right)^{2}\left(\left|\mathbf{v}_{\nu}^{\nu}\right|^{2}-\left|\mathbf{v}_{\nu}^{\mu}\right|^{2}\right)=\mathbf{f}^{\nu} \cdot\left[\left(\mathbf{D}^{\nu \nu}\right)^{2}-\left(\mathbf{D}^{\mu \nu}\right)^{2}\right] \cdot \mathbf{f}^{\nu} \geqslant 0,
$$

and we find that $\left(\mathbf{D}^{\nu \nu}\right)^{2}-\left(\mathbf{D}^{\mu \nu}\right)^{2}$ must also be positive semidefinite. Since $\mathbf{D}^{\mu \nu}=\left(\mathbf{D}^{\nu \mu}\right)^{T}$ through reciprocity of the Green's function, ${ }^{29}$ the hydrodynamic interaction tensor must be chosen such that Eqs. (B5) and (B6) are satisfied simultaneously. There are many ways to do this. For example, one simple scheme is

(1) If Eq. (B5) is not satisfied, set $\mathbf{D}^{\nu \mu}=c \mathbf{D}^{\mu \mu}$, and $\mathbf{D}^{\mu \nu}$ $=\left(\mathbf{D}^{\nu \mu}\right)^{T}$.

(2) If Eq. (B6) is not satisfied, set $\mathbf{D}^{\mu \nu}=c \mathbf{D}^{\nu \nu}$, and $\mathbf{D}^{\nu \mu}$ $=\left(\mathbf{D}^{\mu \nu}\right)^{T}$.

(3) Recheck Eq. (B5). If not satisfied set $\mathbf{D}^{\nu \mu}=c \mathbf{D}^{\nu \mu}$, and $\mathbf{D}^{\mu \nu}=\left(\mathbf{D}^{\nu \mu}\right)^{T}$.

(4) Recheck Eq. (B6). If not satisfied set $\mathbf{D}^{\mu \nu}=c \mathbf{D}^{\mu \nu}$, and $\mathbf{D}^{\nu \mu}=\left(\mathbf{D}^{\mu \nu}\right)^{T}$.

(5) Loop over steps (3) and (4) until Eqs. (B5) and (B6) are both satisfied.

Note that this is a rather general requirement, which means that one should be able to use this with straight OseenBurgers hydrodynamics. This may be advantageous, since there are analytical series solutions for point force hydrodynamics in some geometries. If one were to apply this to point force hydrodynamics, care should be taken that $\mathbf{D}^{\mu \mu}$ also be positive semidefinite for beads very near a surface.

${ }^{1}$ O. S. Andersen, Biophys. J. 77, 2899 (1999).

${ }^{2}$ C. F. Chou, R. H. Austin, O. Bakajin et al., Electrophoresis 21, 81 (2000).

${ }^{3}$ A. Lim, E. T. Dimalanta, K. D. Potamousis et al., Genome Res. 11, 1584 (2001).

${ }^{4}$ N. Perna, G. Plunkett III, V. Burland et al., Nature (London) 409, 529 (2001).

${ }^{5}$ M. Sauer, B. Angerer, W. Ankenbauer et al., J. Biotechnol. 86, 181 (2001).

${ }^{6}$ M. Hinz, S. Gura, B. Nitzan, S. Margel, and H. Seliger, J. Biotechnol. 86, 281 (2001).

${ }^{7}$ R. B. Bird, C. F. Curtiss, R. C. Armstrong, and O. Hassager, Dynamics of Polymeric Liquids (Wiley, New York, 1987), Vol. 2.

${ }^{8}$ M. Fixman, J. Chem. Phys. 69, 1527 (1978).

${ }^{9}$ R. M. Jendrejack, D. C. Schwartz, M. D. Graham, and J. J. de Pablo, J. Chem. Phys. 119, 1165 (2003). 
${ }^{10}$ U. S. Agarwal, A. Dutta, and R. A. Mashelkar, Chem. Eng. Sci. 49, 1693 (1994).

${ }^{11}$ G. Sekhon, R. C. Armstrong, and M. S. Jhon, J. Polym. Sci., Polym. Phys. Ed. 20, 947 (1982).

${ }^{12}$ P. O. Brunn and S. Chi, Rheol. Acta 23, 163 (1984).

${ }^{13}$ P. O. Brunn, J. Chem. Phys. 80, 5821 (1984).

${ }^{14}$ M. S. Jhon and K. F. Freed, J. Polym. Sci., Polym. Phys. Ed. 23, 955 (1985).

${ }^{15}$ X. Fan, N. Phan-Thien, N. T. Yong, X. Wu, and D. Xu, Phys. Fluids 15, 11 (2003).

${ }^{16}$ P. Español and P. Warren, Europhys. Lett. 30, 191 (1995).

${ }^{17}$ M. Ripoll, M. H. Ernst, and P. Español, J. Chem. Phys. 115, 7271 (2001).

${ }^{18}$ R. D. Groot and P. B. Warren, J. Chem. Phys. 107, 4423 (1997).

${ }^{19}$ L. C. Nitsche and E. J. Hinch, J. Fluid Mech. 332, 1 (1997).

${ }^{20}$ R. L. Schiek and E. S. G. Shaqfeh, J. Fluid Mech. 332, 23 (1997).

${ }^{21}$ J. J. de Pablo, H. C. Öttinger, and Y. Rabin, AIChE J. 38, 273 (1992).

${ }^{22}$ L. G. Leal, Annu. Rev. Fluid Mech. 12, 435 (1980).

${ }^{23}$ P. C. H. Chan and L. G. Leal, J. Fluid Mech. 92, 131 (1979).

${ }^{24}$ L. C. Nitsche, AIChE J. 42, 613 (1996).

${ }^{25}$ P. O. Brunn, Int. J. Multiphase Flow 9, 187 (1983).

${ }^{26}$ R. M. Jendrejack, M. D. Graham, and J. J. de Pablo, J. Chem. Phys. 113, 2894 (2000).

${ }^{27}$ R. M. Jendrejack, J. J. de Pablo, and M. D. Graham, J. Chem. Phys. 116, 7752 (2002).

${ }^{28}$ H.-C. Öttinger, Stochastic Processes in Polymeric Fluids (Springer, Berlin, 1996).

${ }^{29}$ C. Pozrikidis, Introduction to Theoretical and Computational Fluid Dynamics (Oxford, New York, 1997).

${ }^{30}$ M. Fixman, Macromolecules 19, 1204 (1986).

${ }^{31}$ R. M. Jendrejack, J. J. de Pablo, and M. D. Graham, J. Non-Newtonian Fluid Mech. 108, 123 (2002).

${ }^{32}$ J. Rotne and S. Prager, J. Chem. Phys. 50, 4831 (1969).

${ }^{33}$ J. F. Marko and E. D. Siggia, Macromolecules 27, 981 (1994).

${ }^{34}$ J. F. Marko and E. D. Siggia, Macromolecules 28, 8759 (1995).
${ }^{35}$ R. M. Jendrejack, J. J. de Pablo, and M. D. Graham, in Technical Proceedings of the Fifth International Conference on Modeling and Simulation of Microsystems (Applied Computational Research Society, San Juan, Puerto Rico, 2002), pp. 88-91.

${ }^{36}$ R. G. Larson, T. T. Perkins, D. E. Smith, and S. Chu, Phys. Rev. E 55, 1794 (1997).

${ }^{37}$ J. S. Hur, E. S. G. Shaqfeh, and R. G. Larson, J. Rheol. 44, 713 (2000).

${ }^{38}$ R. G. Larson, H. Hu, D. E. Smith, and S. Chu, J. Rheol. 43, 267 (1999).

${ }^{39}$ B. Ladoux and P. S. Doyle, Europhys. Lett. 52, 511 (2000).

${ }^{40}$ J. Happel and H. Brenner, Low Reynolds Number Hydrodynamics (Kluwer, Dordrecht, 1991).

${ }^{41}$ C. Pozrikidis, Boundary Integral and Singularity Methods for Linearized Viscous Flow (Cambridge University Press, Cambridge, 1992).

${ }^{42}$ E. R. Dufresne, T. M. Squires, M. P. Brenner, and D. G. Grier, Phys. Rev. Lett. 85, 3317 (2000).

${ }^{43}$ B. U. Felderhof, Physica A 89, 373 (1977).

${ }^{44}$ C. W. J. Beenakker, J. Chem. Phys. 85, 1581 (1986).

${ }^{45}$ K. R. Hase and R. L. Powell, Phys. Fluids 13, 32 (2001).

${ }^{46}$ We used a quadratic velocity, linear pressure formulation. The resultant sparse, symmetric system of equations was solved using GMRES with a partial LU decomposition preconditioner.

${ }^{47} 3 \mathrm{D}$ quadratic interpolation functions are used.

${ }^{48}$ F. M. White, Viscous Fluid Flow (McGraw-Hill, New York, 1991).

${ }^{49}$ M. Daoud and P. G. de Gennes, J. Phys. 38, 85 (1977).

${ }^{50}$ F. Brochard and P. G. de Gennes, J. Chem. Phys. 67, 52 (1977).

${ }^{51}$ F. T. Wall, W. A. Seitz, J. C. Chin, and P. G. de Gennes, Proc. Natl. Acad. Sci. U.S.A. 75, 2069 (1978).

${ }^{52}$ M. Doi and S. F. Edwards, Theory of Polymer Dynamics (Oxford University Press, New York, 1986).

${ }^{53}$ L. Fang, L. Li, H. Hu, and R. G. Larson, Society of Rheology Annual Meeting, Pittsburgh, Oct. 2003.

${ }^{54}$ Y. H. Seo, O. O. Park, and M. S. Chun, J. Chem. Eng. Jpn. 29, 611 (1996).

${ }^{55}$ E. J. Hinch, Phys. Fluids 20, S22 (1977).

${ }^{56}$ G. G. Fuller and L. G. Leal, J. Non-Newtonian Fluid Mech. 8, 271 (1981). 